\title{
Turbidity current with a roof: Success and failure of RANS modeling for turbidity currents under strongly stratified conditions
}

\author{
Tzu-hao Yeh, ${ }^{1,2}$ Mariano Cantero, ${ }^{3}$ Alessandro Cantelli, ${ }^{2}$ Carlos Pirmez, ${ }^{4}$ \\ and Gary Parker ${ }^{1,5}$ \\ Received 11 August 2012; revised 24 July 2013; accepted 9 August 2013.
}

[1] Density underflows in general and turbidity currents in particular differ from rivers in that their governing equations do not allow a steady, streamwise uniform "normal" solution. This is due to the fact that density underflows entrain ambient fluid, thus creating a tendency for underflow discharge to increase downstream. Recently, however, a simplified configuration known as the "turbidity current with a roof" (TCR) has been proposed. The artifice of a roof allows for steady, uniform solutions for flows driven solely by gravity acting on suspended sediment. A recent application of direct numerical simulation (DNS) of the Navier-Stokes equations by Cantero et al. (2009) has revealed that increasing dimensionless sediment fall velocity increases flow stratification, resulting in a damping of the turbulence. When the dimensionless fall velocity is increased beyond a threshold value, near-bed turbulence collapses. Here we use the DNS results as a means of testing the ability of three Reynolds-averaged Navier-Stokes (RANS) models of turbulent flow to capture stratification effects in the TCR. Results showed that the Mellor-Yamada and quasi-equilibrium k- $\varepsilon$ models are able to adequately capture the characteristics of the flow under conditions of relatively modest stratification, whereas the standard k- $\varepsilon$ model is a relatively poor predictor of turbulence characteristics. As stratification strengthens, however, the deviation of all RANS models from the DNS results increases. All are incapable of predicting the collapse of near-bed turbulence predicted by DNS under conditions of strong stratification. This deficiency is likely due to the inability of RANS models to replace viscous dissipation of turbulent energy with transfer to internal waves under conditions of strong stratification. Within the limits of modest stratification, the quasi-equilibrium $\mathrm{k}-\varepsilon$ model is used to derive predictors of flow which can be incorporated into simpler, layer-averaged models of turbidity currents.

Citation: Yeh, T., M. Cantero, A. Cantelli, C. Pirmez, and G. Parker (2013), Turbidity current with a roof: Success and failure of RANS modeling for turbidity currents under strongly stratified conditions, J. Geophys. Res. Earth Surf., 118, doi:10.1002/jgrf.20126.

\section{Introduction}

\subsection{Background}

[2] Turbidity currents are sediment-laden, gravity-driven underflows that occur in lakes and oceans. They are responsible for delivering sediment into these subaqueous

\footnotetext{
${ }^{1}$ Department of Civil and Environmental Engineering, University of Illinois at Urbana-Champaign, Urbana, Illinois, USA.

${ }^{2}$ Shell International Exploration and Production, Houston, Texas, USA.

${ }^{3}$ National Council for Scientific and Technological Research, Institute Balseiro, San Carlos de Bariloche, Argentina.

${ }^{4}$ Shell Nigeria Exploration and Production, Lagos, Nigeria.

${ }^{5}$ Department of Geology, University of Illinois at Urbana-Champaign, Urbana, Illinois, USA.

Corresponding author: T. Yeh, Shell International Exploration and Production, 3333 Hwy 6 South, Houston, TX 77082, USA. (tzu.hao.yeh@gmail.com)

(C)2013. American Geophysical Union. All Rights Reserved. 2169-9003/13/10.1002/jgrf.20126
}

environments, where they can shape the largest sedimentary features on the modern earth [Middleton, 1993]. Complex interactions between turbidity currents and their surrounding environment take place as they travel and evolve. First of all, the difference in density between the turbidity current and the ambient fluid causes interfacial instability, which acts to entrain ambient fluid into the current body. As a result, the current thickens and becomes dilute. Second, these currents actively exchange sediment with the bed surface material by depositing sediment through downward settling and entraining sediment through shear, creating depositional or erosional features in the process. These interactions are keys to explaining the formation of important subaqueous morphologies such as submarine channels, levees, and canyons.

[3] One of the most important factors associated with these interactions is the internal structure of the current. For example, the near-bed velocity gradient and the turbulent energy determine the erosive power of a current; the near-bed sediment concentration determines settling flux onto the bed. On the other hand, as the current evolves, settling and entrainment 
processes will in turn adjust its internal structure. Such a system can be further complicated when the dispersive component, sediment in our case, self-stratifies. Due to the tendency for sediment to settle, the concentration tends to decrease upward within the current body. This stratification in density requires additional consumption of energy from the mean flow in order to mix the concentration against the density gradient. As turbulence dampens, the flow becomes less effective at mixing momentum, resulting in a velocity profile that varies more strongly in the vertical [Vanoni, 1946; Einstein and Chien, 1955; Coleman, 1981, 1986; Lyn 1988].

[4] Various efforts have been devoted to understanding the hydrodynamics as well as the morphodynamic response associated with turbidity currents through numerical modeling. These studies are often composed of a depth-averaged, three-equation model [Ellison and Turner, 1959; Pantin, 1979] for flow dynamics and an equation for bed movement. For example, Imran et al. [1998] examined the condition for self-channelization of turbidity currents on a submarine fan using a two-dimensional three-equation model; Kostic and Parker [2003] implemented the model as a submodel to study the depositional pattern of fine material beyond a prograding delta foreset; Bradford and Katopodes [1999] extended the model to include multiple-grain sizes; Parker et al. [1986] included an additional equation for the balance of turbulent kinetic energy, resulting in a four-equation model, to restrain unrealistic self-acceleration of turbidity currents that appeared in the three-equation model. While these depth-averaged models are more computationally efficient and are often applied to large-scale simulations, various empirical relations and/or assumptions are required to close the problem, thus compromising the accuracy of the models. An example is the use of a specified, constant near-bed concentration ratio, $r_{0}=c_{b} / C$, where $c_{b}$ and $C$ are the near-bed and depth-averaged volumetric sediment concentration, respectively. Such an assumption is intuitively inaccurate since the value of $r_{0}$ should vary at least with the grain size in the flow. More specifically, for the same layer-averaged concentration, an increase in sediment size should result in a higher near-bed concentration as the particles become increasingly difficult to keep in suspension in the flow. Similar arguments can be made in regard to the use of a constant resistant coefficient $C_{f}$ when computing the bed shear stress.

[5] Finding suitable relations for these parameters, however, has been challenging. Field observations in the deep water environment have recently become feasible [Xu et al., 2004; Xu, 2010] but are nevertheless difficult to obtain due to the unpredictable nature of turbidity currents and the depth at which they often occur. Often, the evidence of an event is only retrieved from the resulting deposit, i.e., turbidites, as observed in surface expression cores or seismics [e.g., Babonneau et al., 2002; Fildani et al., 2006], or in outcrop [e.g., Ito and Saito, 2006]. On the other hand, turbidity currents in the laboratory can be produced and recorded under more controlled conditions. However, due to scale effects, viscous effects tend to be overemphasized in laboratory turbidity currents and hence limitations arise in regard to application of the results at field scale.

[6] Several numerical efforts have attempted to address this issue by using depth-resolving Reynolds-averaged Navier-Stokes (RANS) models to capture certain level of details of turbulence, the vertical structure of the flow, and hence reduce empiricism [e.g., Huang et al., 2004; Choi and Garcia, 2002]. These models, when coupled with an equation that accounts for mass conservation of the bed, serve as a good tool for understanding and predicting the morphodynamic responses in the deep water environment. For example, Khan and Imran [2008] used a one-equation turbulence closure with the Exner equation to investigate the flow characteristics and the filling process of minibasins by turbidity currents on the continental slope; Abd El-Gawad et al. [2012a, 2012b] implemented the Mellor-Yamada model at a field scale to examine the flow and depositional pattern in a submarine-meandering channel.

[7] The stratification effects in these depth-resolving RANS models are typically incorporated using a buoyancy production term which, under stably stratified conditions, acts to damp turbulent kinetic energy and reduce mixing. While these models have achieved good results compared to laboratory data and been widely applied to field-scale simulations, the limitations to such an approach in capturing the stratification effects have not been examined in detail. As a result, finetuning of some parameters cannot be avoided in general to ensure the success of such models. Moreover, the results obtained from these models are usually problem-specific, varying according to boundary and initial conditions. Hence, they are not readily used to generate physically based rules that enhance our basic knowledge on the nature of the flow or improve modeling efficiency in large-scale simulations.

\subsection{Turbidity Current With a Roof}

[8] The concept of a turbidity current with a roof (TCR) proposed by Cantero et al. [2009], hereinafter noted as $\mathrm{C} 09$, is designed to provide this basic insight. A recapitulation of their work is given as follows. The configuration for TCR is shown in Figure 1. The tank is filled with fresh water and, in the absence of sediment, the ambient fluid in the channel is still and the local pressure is hydrostatic. Upon the release of the sediment at the upstream end of the channel, gravity drives the sediment to form a turbidity current which travels down the slope. The sediment and water are free to mix, but only within the channel. The roof of the channel prevents further entrainment of the ambient water into the channel, and hence sets a maximum thickness of the flow (i.e., roof height). Under appropriate conditions, the nearbed downward (depositional) sediment flux associated with sediment fall velocity can be fully compensated for by resuspension into the flow by turbulence, yielding zero net sediment deposition on the channel bed.

[9] The flow in the channel is treated as a single-phase fluid where the suspended particles are assumed to follow closely the turbulence movement up to a fall velocity. In reality, this is true only when the size of the particles is small compared to the Kolmogorov scale of turbulence. For larger particles, separation between the particles and the fluid may lead to vortex shedding and enhance turbulence [Gore and Crowe, 1991; Nino and Garcia, 1998]. In C09 and the present work, however, such effects are neglected regardless of the flow conditions in order to investigate only the role of density stratification on turbulence attenuation.

[10] In C09, the details of a rough boundary are not modeled. The details of the region at the interface between the bed and the flow are replaced with a thin molecular sublayer, in which sediment can be sequestered. This is 


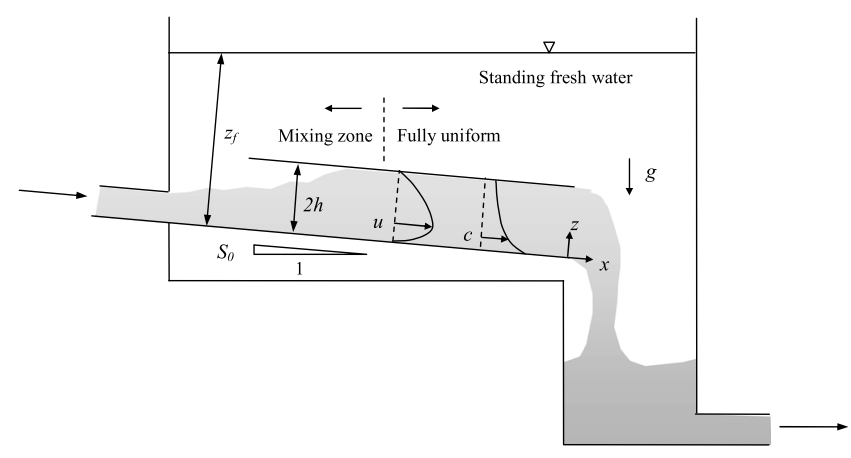

Figure 1. Configuration for turbidity current with a roof (TCR). A turbidity current driven by the excess density of the water-sediment mixture evolves in the channel. By limiting water entrainment and imposing complete sediment bypass conditions, an idealized normal flow condition can be achieved in the channel. The diagram has been redrawn from Cantero et al. [2009].

achieved by introducing an artificial "molecular" diffusivity of sediment concentration. In this configuration, sediment is deposited into, and entrained by turbulence from, this thin "molecular" layer rather than the bed itself.

[11] In such a configuration, the turbidity current in the channel can eventually reach normal (steady and streamwise uniform when averaged over turbulence) conditions either sufficiently far downstream or after a sufficient period of time has passed. By focusing on this normal flow condition, which is made possible by the presence of a roof, we can understand the behavior, and in particular, that associated with sediment stratification effects, of turbidity currents in a way that allows extraction of useful generalities and information/rules. Due to the presence of the roof, TCR does not give a precise model of what may be found in natural turbidity currents. The results of TCR are, however not problem-specific, but rather dependent only on physically based dimensionless parameters.

[12] In this study, we investigate the performance and limitations of RANS models in characterizing turbidity currents under the setting of a TCR. Three of most widely implemented turbulence closures for stratified flows are used here: standard k- $\varepsilon$ [Rodi, 1993], Mellor-Yamada [Mellor and Yamada, 1974, 1982] (referred to as M-Y hereinafter), and quasi-equilibrium $\mathrm{k}-\varepsilon$ [Burchard et al., 1998] (referred to as QE k- $\varepsilon$ hereinafter). We examine the capability of these models to reproduce the mean flow and turbulence fields given by the direct numerical simulation (DNS) results from C09. It is found that all three models can capture the stratification effects, but only up to a certain threshold. Two failings common to the RANS models used in this study are identified. First of all, the "fish trap" effect manifested in the sharp density gradient near the velocity maximum separates the sediment concentration into two distinct regions. Such a phenomenon is associated with the overemphasized reduction of the eddy diffusivity caused by the structure of the closures. The artificial sequestration of the sediment in the near-bed region also implies errors when using these models for large-scale flow and morphodynamic predictions.

[13] If the flow is stratified, a portion of the energy is also transferred to and dissipated in the form of the internal waves. Such a process is typically described by wave-wave theory [Gregg, 1989; Müller et al., 1986], which is fully independent from the classical turbulence energy cascade theory. For weakly stratified shear flows like most natural turbidity currents, turbulence dominates and the presence of internal waves has rarely been considered in the literature as an additional energy sink. Under strongly stratified conditions, however, a treatment of the wave energy has to be incorporated to account for the additional energy loss. This leads to the second failing of the RANS models. It is found that beyond a criterion where flow relaminarization occurs, significant errors arise in all RANS model predictions, due to both inappropriate specification of boundary conditions and the inability of RANS closures to capture the level of turbulence damping. By examining the various length scales associated with turbulence and buoyancy, we relate such failure to the inability of the RANS models to describe energy dissipation due to internal waves.

[14] The three models examined in this study represent a small but representative (due to their popularity) subset of a variety of second-moment turbulence closures. More recently, the standard $\mathrm{k}-\varepsilon$ model has been criticized for a degree of lack of physical soundness, in that it uses small-scale turbulence to determine the macroscale of turbulence [Mellor and Yamada, 1982; Kantha, 2004]. The M-Y model rectified this by introducing an equation for a macroscale length. In addition to the differences in the length scale equations, the role of stability functions is also crucial. We show that the models which incorporate stability functions in the calculation of the eddy viscosity and eddy diffusivity, such as the M-Y and QE k- $\varepsilon$ models, outperform the standard k- $\varepsilon$ model in terms of agreement with the DNS results.

[15] The rest of the paper is laid out as follows. In section 2 , we introduce the governing equations and turbulence closures. We also derive and examine the boundary conditions in our models in detail. In section 3, we review some of the flow settings and important findings of C09 which set the foundation for our model comparison. In section 4, numerical methods and model validation using two special cases are presented. Results for Regime I (below the threshold for the failure of RANS models) and Regime II flow conditions (above this threshold, as defined in section 3) are presented and discussed in sections 5 and 6, respectively. In section 7 , we apply the model to examine the sensitivity in flow characteristics to variation in two important dimensionless parameters, the dimensionless settling velocity and shear Richardson number. The effect of multiple-grain sizes on 
the mean flow and turbulent field is also examined. A summary and the conclusions of this study are given in section 8 .

\section{Problem Formulation}

\subsection{Governing Equations}

[16] The problem can be described in terms of standard Reynolds-averaged Navier-Stokes (RANS) equations for incompressible flow, with an additional consideration for the change in density due to suspended sediment. For flows with dilute suspensions, this effect can be approximated with additional gravity terms in the momentum equations associated with the Boussinesq approximation. In the cross-channel direction $y$, we assume that all flow characteristics are uniform, i.e., $\partial() / \partial y=0$, and the Reynolds-average velocity $v=0$. The simplified RANS equations are given by

$$
\begin{aligned}
\frac{\partial \rho_{a}}{\partial t}+ & \rho_{a}\left(\frac{\partial u}{\partial x}+\frac{\partial w}{\partial z}\right)=0 \\
\frac{\partial u}{\partial t}+u \frac{\partial u}{\partial x}+w \frac{\partial u}{\partial z}= & -\frac{1}{\rho_{a}} \frac{\partial p}{\partial x}+v\left(\frac{\partial^{2} u}{\partial x^{2}}+\frac{\partial^{2} u}{\partial z^{2}}\right) \\
& -\frac{1}{\rho_{a}}\left(\frac{\partial\left(\rho_{a} \overline{u^{\prime 2}}\right)}{\partial x}+\frac{\partial\left(\rho_{a} \overline{u^{\prime} w^{\prime}}\right)}{\partial z}\right) \\
& +(1+R c) g S_{0}, \\
\frac{\partial w}{\partial t}+u \frac{\partial w}{\partial x}+w \frac{\partial w}{\partial z}= & -\frac{1}{\rho_{a}} \frac{\partial p}{\partial z}+v\left(\frac{\partial^{2} w}{\partial x^{2}}+\frac{\partial^{2} w}{\partial z^{2}}\right) \\
& -\frac{1}{\rho_{a}}\left(\frac{\partial\left(\rho_{a} \overline{u^{\prime} w^{\prime}}\right)}{\partial x}+\frac{\partial\left(\rho_{a} \overline{w^{\prime 2}}\right)}{\partial z}\right) \\
& +(1+R c) g
\end{aligned}
$$

where $t$ is time; $(x, z)$ are the streamwise and upward normal coordinates, respectively; $(u, w)$ are the Reynolds-averaged velocities in the $(x, z)$ direction; $\rho_{a}$ and $\rho_{s}$ are densities of the ambient fluid and sediment, respectively; $p$ is Reynoldsaveraged total pressure; $v$ is kinematic viscosity; $-\rho_{a} \overline{u^{\prime 2}},-\rho_{a}$ $\overline{w^{\prime 2}}$, and $-\rho_{a} \overline{u^{\prime} w^{\prime}}$ are Reynolds stresses associated with instantaneous fluctuations in streamwise and upward normal velocities $\left(u^{\prime}, w^{\prime}\right) ; c$ is Reynolds-averaged total sediment volume concentration; $R=\left(\rho_{s}-\rho_{a}\right) / \rho_{a}$ (=1.65 for quartz), $g$ is gravitational acceleration, and $S_{0}$ is channel slope. In the case where the suspension contains $N$ size fraction ranges, $c=\sum_{i=1}^{N} c_{i}$, where $c_{i}$ denotes the volume concentration in the $i$ th fraction range.

[17] Under steady, uniform flow and no-slip boundary conditions, equation (1) gives $w=0$. As a result, the momentum equations simplify to

$$
\begin{gathered}
\frac{\partial u}{\partial t}=v \frac{\partial^{2} u}{\partial z^{2}}-\frac{1}{\rho_{a}} \frac{\partial\left(\rho_{a} \overline{u^{\prime} w^{\prime}}\right)}{\partial z}+R c g S_{0} \\
\left.p=\rho_{a} g\left(z_{f}-z+R\right]_{z}^{2 h} c \mathrm{~d} z\right)
\end{gathered}
$$

where $z_{f}=z_{f}(x)$ is the free surface elevation and $2 h$ is the gap height of the roof, as shown in Figure 1. Equation (5) describes the total pressure as the sum of the hydrostatic pressure and an added pressure due to the overlying submerged weight of the suspended sediment. The hydrostatic part of the total pressure, when introduced into equation (2), results in a pressure gradient which balances out the streamwise gravitational force induced by the ambient fluid, and the remaining pressure gradient associated with suspended sediment vanishes under the uniform flow assumption. The sediment concentration in this study is considered to be diluted and the particles in suspension are assumed to be sufficiently small that they follow closely the water movement up to a settling velocity. Under these assumptions, the sediment concentration is governed by the following conservation equation:

$$
\frac{\partial c_{i}}{\partial t}-v_{\mathrm{si}} \frac{\partial c_{i}}{\partial z}=\frac{v}{S c} \frac{\partial^{2} c_{i}}{\partial z^{2}}-\frac{\partial \overline{w^{\prime} c_{i}^{\prime}}}{\partial z}, \quad i=1 \sim N,
$$

where $v_{\mathrm{si}}$ is the settling velocity for that range, $c^{\prime}{ }_{i}$ is the instantaneous fluctuating part of concentration, and $-\overline{w^{\prime} c^{\prime}}$ is the Reynolds flux of that range. The Schmidt number is defined as $S c=v / v_{c}, v_{c}$ being the artificial molecular diffusivity of sediment concentration as explained above.

[18] Now, let $\tau_{b}$ denote the mean shear stress on the bed $(z=0)$ and $\tau_{t}$ define the corresponding shear stress on the roof $(z=2 h)$, both signed so that they act to resist the downslope pull of gravity. Integration of equation (4) in $z$ for steady, uniform flow yields the following result for mean shear stress $\tau$ :

$$
\tau \equiv \rho_{a}\left(v \frac{\mathrm{d} u}{\mathrm{~d} z}-\overline{u^{\prime} w^{\prime}}\right)=-\tau_{t}+\rho_{a} R g S_{0} \int_{z}^{2 h} c \mathrm{~d} z .
$$

[19] Evaluating equation (7) at $z=0$ gives

$$
u_{, \text {avg }}=\sqrt{R C g h S_{0}},
$$

where $C$ is the total concentration averaged over $2 h$, and $u_{*}$,avg is a nominal shear velocity given as

$$
u_{*_{, \text {avg }}}^{2}=\frac{1}{2}\left(u_{*_{, t}}^{2}+u_{*_{, b}}^{2}\right),
$$

where the shear velocities $u_{*, b}$ and $u_{*, t}$ at the bed and roof are related to the corresponding shear stresses $\tau_{b}$ and $\tau_{t}$ as

$$
\tau_{b}=\rho_{a} u_{*, b}^{2}, \tau_{t}=\rho_{a} u_{*, t}^{2}
$$

[20] The governing equations (4) and (6) are made dimensionless using $h$ and $u_{* \text {,avg }}$; in addition, concentration is normalized such that $\hat{c}_{i}=c_{i} / C$, giving

$$
\begin{gathered}
\frac{\partial \hat{u}}{\partial \hat{t}}=\frac{\partial}{\partial \hat{z}}\left(\frac{1}{R e_{\tau}} \frac{\partial \hat{u}}{\partial \hat{z}}-\widehat{\widehat{u^{\prime} w^{\prime}}}\right)+\hat{c}, \\
\frac{\partial \hat{c}_{i}}{\partial \hat{t}}-\hat{v}_{\mathrm{si}} \frac{\partial \hat{c}_{i}}{\partial \hat{z}}=\frac{\partial}{\partial \hat{z}}\left(-\widehat{\widehat{w^{\prime} c^{\prime}}}+\frac{1}{\operatorname{Re}_{\tau} S c} \frac{\partial \hat{c}_{i}}{\partial \hat{z}}\right), \quad i=1 \sim N,
\end{gathered}
$$

where the hats denote dimensionless parameters, $\hat{c}=\sum_{N} \hat{c}_{i}$ and

$$
R e_{\tau}=\frac{u *_{\text {,avg }} h}{v}
$$

denotes the shear Reynolds number. This parameter characterizes the state of development of the turbulence of the flow.

\subsection{Turbulence Closures}

[21] The Reynolds stress and flux terms are modeled using eddy diffusivities. In dimensionless forms, these are given by 


$$
\begin{gathered}
\widehat{\widehat{u^{\prime} w^{\prime}}}=-\hat{v}_{t} \frac{\partial \hat{u}}{\partial \hat{z}}, \\
\widehat{\widehat{w^{\prime} c_{i}^{\prime}}}=-\hat{v}_{t c} \frac{\partial \hat{c}_{i}}{\partial \hat{z}}, \quad i=1 \sim N,
\end{gathered}
$$

where $\hat{v}_{t}$ and $\hat{v}_{\text {tc }}$ are the eddy viscosity (eddy diffusivity of momentum) and eddy diffusivity of sediment concentration, respectively. Summing over all $N$ size fractions, the total Reynolds flux is given by

$$
\widehat{\overline{w^{\prime} c^{\prime}}}=\sum_{i=1}^{N} \widehat{\widehat{w^{\prime} c_{i}^{\prime}}}=-\hat{v}_{t c} \frac{\partial \hat{c}}{\partial \hat{z}} .
$$

[22] In this study, we model $\hat{v}_{t}$ and $\hat{v}_{\text {tc }}$ using three differential models: standard k- $\varepsilon$ [Rodi, 1993], level 2-1/2 [Mellor and Yamada, 1974, 1982], and quasi-equilibrium k- $\varepsilon$ [Burchard et al., 1998]. The formulations and detailed description can be found in the corresponding literature. In the following, we present the basic equations involved in these models and the choice of coefficients.

\subsubsection{Mellor-Yamada (M-Y)}

[23] At level 2-1/2, the M-Y model assumes local equilibrium for all turbulent characteristics except for turbulent kinetic energy $k$. It also introduces an empirically based equation to govern the variation of a master length scale used in the relation for eddy viscosity. In dimensionless form, these read

$$
\begin{aligned}
\frac{\partial \hat{q}^{2}}{\partial \hat{t}} & =\frac{\partial}{\partial \hat{z}}\left(\left(S_{q} \hat{q} \hat{l}+\frac{1}{R e_{\tau}}\right) \frac{\partial \hat{q}^{2}}{\partial \hat{z}}\right)+2 \hat{P}+2 \hat{B}-2 \hat{\varepsilon} \\
\frac{\partial \hat{q}^{2} \hat{l}}{\partial \hat{t}}= & \frac{\partial}{\partial \hat{z}}\left(\left(S_{l} \hat{q} \hat{l}+\frac{1}{R e_{\tau}}\right) \frac{\partial \hat{q}^{2} \hat{l}}{\partial \hat{z}}\right)+\hat{l}\left(E_{1} \hat{P}+E_{3} \hat{B}\right) \\
& -\frac{\hat{q}^{3}}{B_{1}}\left(1+E_{2}\left(\frac{\hat{l}}{\kappa \hat{L}}\right)^{2}\right)
\end{aligned}
$$

where $\hat{q}^{2}=\widehat{u^{\prime 2}}+\widehat{v^{\prime 2}}+\widehat{w^{\prime 2}}$ is twice the turbulent kinetic energy per unit mass; $\hat{l}$ is the master length scale; and $\hat{L}$ is a measure of the distance from the wall which takes the form $\hat{L}=\max (\hat{z}, 2-\hat{z})$ for the configuration in question. In addition, $S_{q}, S_{l}, E_{1}, E_{2}, E_{3}$, and $B_{1}$ are empirical coefficients given in Table 1 , and $\kappa$ denotes the von Kármán constant. At steady state, equation (17) describes the balance of energy as governed by, beginning from the leftmost term, the energy transport rate by diffusion, energy production rate $\hat{P}$, buoyancy production rate $\hat{B}$, and energy dissipation rate $\hat{\varepsilon}$. The last three terms are given by

$$
\begin{gathered}
\hat{P}=-\widehat{\overline{u^{\prime} w^{\prime}}}\left(\frac{\partial \hat{u}}{\partial \hat{z}}\right), \\
\hat{B}=-R i_{\tau} \widehat{\widehat{w^{\prime} c^{\prime}}}, \\
\hat{\varepsilon}=\frac{\hat{q}^{3}}{B_{1} \hat{l}}
\end{gathered}
$$

where

$$
R i_{\tau}=\frac{R C g h}{u_{*_{\text {,avg }}}^{2}}
$$

is the shear Richardson number, which characterizes the degree of stratification. In stably stratified flows, $\hat{B}<0$, indicating that turbulent kinetic energy is consumed in order to mix suspended sediment against the density gradient.

[24] For specified values of $\hat{q}$ and $\hat{l}$, the eddy viscosity and eddy diffusivity are calculated using the relations

$$
\begin{aligned}
& \hat{v}_{t}=\hat{l} \hat{q} S_{M}, \\
& \hat{v}_{t c}=\hat{l} \hat{q} S_{H},
\end{aligned}
$$

where $S_{M}$ and $S_{H}$ are stability functions given by [Galperin et al., 1988]

$$
\begin{gathered}
S_{M}=\frac{A_{1}\left[\left(1-\frac{6 A_{1}}{B_{1}}-3 C_{1}\right)-3 A_{2} G_{H}\left(\left(B_{2}-3 A_{2}\right)\left(1-\frac{6 A_{1}}{B_{1}}\right)-3 C_{1}\left(6 A_{1}+B_{2}\right)\right)\right]}{\left[\left(1-9 G_{H} A_{1} A_{2}\right)\left(1-3 A_{2}\left(6 A_{1}+B_{2}\right) G_{H}\right)\right]} \\
S_{H}=\frac{A_{2}\left(1-\frac{6 A_{1}}{B_{1}}\right)}{1-3 A_{2} G_{H}\left(6 A_{1}+B_{2}\right)}
\end{gathered}
$$

and

$$
G_{H}=R i_{\tau}\left(\frac{\hat{l}}{\hat{q}}\right)^{2} \frac{\partial \hat{c}}{\partial \hat{z}}
$$

[25] In the above relations, $A_{1}, A_{2}$, and $B_{2}$ are empirical constants given in Table 1.

[26] The flow is stably stratified when $\partial \hat{c} / \partial \hat{z} \leq 0$. In applying $\mathrm{M}-\mathrm{Y}$ to stable stratification, however, the constraint $G_{H}>-0.28$ is imposed [Galperin et al., 1988]. For values of $G_{H}$ below this threshold, turbulent energy is converted to the energy of internal waves. We will discuss this in detail in section 6 .

[27] As noted above, the values of the coefficients used in this study are listed in Table 1. The only difference from those given by Mellor and Yamada [1982] is the value of $E_{3}$, which they set equal to $E_{1}$. Their choice, however, has been shown in previous studies to prevent homogeneous shear flows from reaching steady state [Baumert and Peters, 2000; Burchard, 2001]. Here we adopt the value derived by Burchard [2001] under the above constraint and set $E_{3}=5.093$. The dimensionless parameter $S c_{t}$ in $A_{2}$, is the turbulent counterpart of the Schmidt number, defined as $S c_{t}=\hat{v}_{t 0} / \hat{v}_{t c 0}$, where $\hat{v}_{t 0}$ and $\hat{v}_{t c 0}$ are eddy viscosity and eddy diffusivity under neutral conditions, respectively. In the absence of density gradient, equation (26) show that the ratio of $\hat{v}_{t}$ to $\hat{v}_{t c}$ is equal to $S c_{t}$ at any $\hat{z}$. Such invariance, however, does not hold when the flow becomes stratified.

\subsubsection{Standard $\mathbf{k}-\boldsymbol{\varepsilon}$}

[28] This model consists of two additional equations beyond mass and momentum balance, which correspond to the conservation of the turbulent kinetic energy $k$ and energy dissipation $\varepsilon$ :

$$
\frac{\partial \hat{k}}{\partial \hat{t}}=\frac{\partial}{\partial \hat{z}}\left(\left(\frac{\hat{v}_{t}}{\sigma_{k}}+\frac{1}{R e_{\tau}}\right) \frac{\partial \hat{k}}{\partial \hat{z}}\right)+\hat{P}+\hat{B}-\hat{\varepsilon}
$$

$$
\frac{\partial \hat{\varepsilon}}{\partial \hat{t}}=\frac{\partial}{\partial \hat{z}}\left(\left(\frac{\hat{v}_{t}}{\sigma_{\varepsilon}}+\frac{1}{R e_{\tau}}\right) \frac{\partial \hat{\varepsilon}}{\partial \hat{z}}\right)+C_{\varepsilon 1} \frac{\hat{\varepsilon}}{\hat{k}}\left(\hat{P}+C_{\varepsilon 3} \hat{B}\right)-C_{\varepsilon 2} \frac{\hat{\varepsilon}^{2}}{\hat{k}} .
$$

[29] The eddy viscosity and eddy diffusivity are calculated by 
Table 1. Coefficients for the M-Y Model

\begin{tabular}{lc}
\hline M-Y Models & Coefficients \\
\hline$A 1$ & 0.92 \\
$A 2$ & $A_{1}\left(\gamma_{1}-C_{1}\right) /\left(\gamma_{1} S c_{t}\right)$ \\
$B_{1}$ & 16.6 \\
$B_{2}$ & 10.1 \\
$C_{1}$ & 0.08 \\
$E_{1}$ & 1.8 \\
$E_{2}$ & 1.33 \\
$E_{3}$ & 5.093 \\
$\gamma_{1}$ & 0.22 \\
$S_{q}$ & 0.2 \\
$S_{l}$ & 0.2 \\
\hline
\end{tabular}

$$
\hat{v}_{t}=C_{\mu} \frac{\hat{k}^{2}}{\hat{\varepsilon}}
$$

and

$$
\hat{v}_{t c}=C_{\mu c} \frac{\hat{k}^{2}}{\hat{\varepsilon}} .
$$

[30] In the standard k- $\varepsilon$ model, the values for empirical coefficients $\sigma_{k}, \sigma_{\varepsilon}, C_{\varepsilon 1}$, and $C_{\varepsilon 2}$ (listed in Table 2) are pure constants calibrated from flows under neutral conditions [Rodi, 1993] and $C_{\mu c}=C_{\mu} / S c_{t}$. Rodi [1993] suggested that the value of $C_{\varepsilon 3}$ ranges between 0 and 1, where 0 corresponds to a horizontal flow.

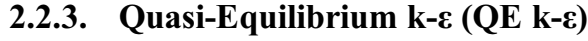

[31] Following Burchard et al. [1998], we express equations (30) and (31) in alternative forms using the Kolmogorov-Prandtl relation

$$
\hat{v}_{t}=c_{\mu} \hat{k}^{1 / 2} \hat{l}
$$

and

$$
\hat{v}_{\mathrm{tc}}=c_{\mu c} \hat{k}^{1 / 2} \hat{l}
$$

where $c_{\mu}$ and $c_{\mu c}$ stability functions resembling $S_{M}$ and $S_{H}$, respectively, in the M-Y model. The full dependency of $c_{\mu}$ are $c_{\mu c}$ on dimensionless turbulent shear number and turbulent buoyancy number can be found in Burchard and Baumert [1995] and Luyten et al. [1996]. For the sake of simplicity and consistency, we assume these functions directly relate to $S_{M}$ and $S_{H}$ according to the relations $c_{\mu}=\sqrt{2} S_{M}$ and $c_{\mu c}=\sqrt{2} S_{H}$. Under the Kolmogorov hypothesis of local, small-scale isotropy, the energy dissipation rate is modeled by

$$
\hat{\varepsilon}=\left(c_{\mu}^{0}\right)^{3} \frac{\hat{k}^{3 / 2}}{\hat{l}},
$$

where $c_{\mu}^{0}$ is pure constant equal to $c_{\mu}$ in the absence of stratification effect, i.e., $c_{\mu}^{0}=3 \sqrt{2} A_{1}\left(\gamma_{1}-C_{1}\right) \approx 0.5465$. Substituting equation (34) into (32) and (33) and comparing with equations (30) and (31), we get

$$
C_{\mu}=\left(c_{\mu}^{0}\right)^{3} c_{\mu}
$$

and

$$
C_{\mu c}=\left(c_{\mu}^{0}\right)^{3} c_{\mu c}
$$

[32] It can be shown that in the absence of stratification effects, $C_{\mu}=\left(c_{\mu}^{0}\right)^{4} \approx 0.09$, a value identical to that in the standard k- $\varepsilon$ model.

\subsection{Boundary Conditions}

[33] The first question regarding the boundary conditions is where they should be applied. For most of the existing RANS models, the closures do not describe the turbulence field well into the viscous sublayer at the vicinity of the wall. Instead, these models usually choose a reference distance $b$ away from the wall and apply boundary conditions according to, for example, the logarithmic law. In order to minimize contamination from the viscous sublayer, this reference distance should be sufficiently far away from the wall. For typical wall flows, at least under neutral conditions, the criterion $z^{+}=\left(\operatorname{Re}_{\tau} \hat{b}\right)>50$, where $\hat{b}=b / h$, diminishes the viscous influence down to less than 10\% [Pope, 2000] and can be used for specifying $\hat{b}$. However, this criterion is inappropriate in the context of comparing with the DNS results from C09. Due to the low value of $R e_{\tau}$ used in C09 (a constraint imposed by the limitations of DNS), applying the criterion would compromise a significant portion of the computational domain (more than 25\%). More importantly, as more sediment suspension concentrates toward the bed with increasing value of $\hat{v}_{s}$, the choice of large $\hat{b}$ cannot capture the sediment concentration in that region and hence the driving force of the flow within it. It should be noted that the choice of large $\hat{b}$ is much less problematic in open channel flows carrying dilute suspensions, because the gravitational force acting on the fluid, rather than the sediment, comprises the dominant driving force. In this study, we set $\hat{b}$ to twice the thickness of the viscous sublayer, i.e., $\hat{b}=23.2 / R e_{\tau} \approx 0.129$. While a small region close to the walls may still be affected by the viscous effects, the resulting velocity and turbulent characteristics at the center and major portion of the channel are in good agreement with those from DNS under neutral conditions (see section 4).

[34] The boundary conditions for the flow velocity are given by the logarithmic law as

$$
\begin{aligned}
& \hat{u}_{b}=\left.\hat{u}\right|_{\hat{z}=\hat{b}}=\frac{\hat{u} *_{, b}}{\kappa} \ln \left(\operatorname{Re}_{\tau} \hat{u} *_{, b} \hat{b}\right)+5.5 \hat{u} *_{, b}, \\
& \hat{u}_{t}=\left.\hat{u}\right|_{\hat{z}=2-\hat{b}}=\frac{\hat{u} *, t}{\kappa} \ln \left(\operatorname{Re}_{\tau} \hat{u} *_{, t} \hat{b}\right)+5.5 \hat{u} *_{, t},
\end{aligned}
$$

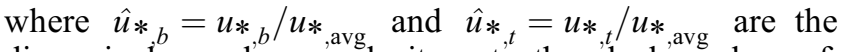
dimensionless shear 'velocity at the 'bed 'and roof, respectively. From the momentum balance described by equations (8) and (9), it is clear that the sum of the two shear stresses should be equivalent to the total driving force, i.e., $u_{*, b}^{2}+u_{*, t}^{2}=2 R C g h S_{0}=2 u_{*, \text { avg }}^{2}$, or in dimensionless form,'

$$
\hat{u}_{*, b}^{2}+\hat{u}_{*, t}^{2}=2 .
$$

[35] The velocities at the boundaries remain indeterminate without additional relations for $\hat{u}_{*_{,},}$and $\hat{u} *_{, t}$. To derive these relations, we rewrite equation (11) at steady state, substituting the Reynolds stress using equation (14), and integrate at the near-wall region to get

$$
\begin{gathered}
\left.\left(\left(\hat{v}_{t}+\frac{1}{R e_{\tau}}\right) \frac{\partial \hat{u}}{\partial \hat{z}}\right)\right|_{\hat{z}=\hat{b}}=\hat{u}_{*, b}^{2}-\int_{0}^{\hat{b}} \hat{c} \mathrm{~d} \hat{z} \approx \hat{u}_{*, b}^{2}-\hat{b} \\
\left.\left(\left(\hat{v}_{t}+\frac{1}{R e_{\tau}}\right) \frac{\partial \hat{u}}{\partial \hat{z}}\right)\right|_{\hat{z}=2-\hat{b}}=-\hat{u}_{*_{, t}}^{2}+\int_{2-\hat{b}}^{2} \hat{c} \mathrm{~d} \hat{z} \approx-\hat{u}_{*_{, t}}^{2}+\hat{b} .
\end{gathered}
$$

[36] The approximations made above are necessitated by the fact that we do not have prior knowledge on the values 
Table 2. Coefficients for the k- $\varepsilon$ Model

\begin{tabular}{lccc}
\hline & Standard & & Quasi-equlibrium \\
\hline$C_{\varepsilon 1}$ & & 1.44 & \\
$C_{\varepsilon 2}$ & & 1.92 & -1.4 \\
$C_{\varepsilon 3}$ & 0 & 1.0 & 1.08 \\
$\sigma_{k}$ & 1.3 & & 0.5465 \\
$\sigma_{\varepsilon}$ & --- & & $\left(c_{\mu}^{0}\right)^{3} c_{\mu}$ \\
$c_{\mu}^{0}$ & 0.09 & & $\left(c_{\mu}^{0}\right)^{3} c_{\mu c}$ \\
$C_{\mu}$ & $C_{\mu} / S c_{t}$ & & \\
$C_{\mu c}$ & & & \\
\hline
\end{tabular}

of the integrals, but justified as long as $\hat{b}$ is sufficiently small. This treatment allows precise prediction of shear stress under neutral conditions but displays increasing error as $\hat{v}_{s}$ increases. In order to illustrate this, it is of value here to show some computational results in advance. Figure 2 shows the differences in the dimensionless total shear stresses at the bed $(\hat{z}=\hat{b})$ and the roof $(\hat{z}=2-\hat{b})$ between those obtained from the above approximations and those using DNS. At the roof, where stratification effects are small in almost all cases (see section 3), approximation (41) works well. On the other hand, near the bed where the stratification effects are much more significant, the approximated values increasingly deviate from DNS with increasing $\hat{v}_{S}$ . However, it can be argued that under moderate stratification effects, these approximations are fairly reasonable. At sufficiently large Reynolds number, the error can be reduced by specifying a smaller $\hat{b}$. With boundary condition (40), we integrate the steady version of equation (11) twice in $\hat{z}$ to obtain

$$
\begin{aligned}
\hat{u}_{t}= & \hat{u}_{b}+\left(\hat{u}_{*_{, b}}^{2}-\hat{b}\right) \int_{\hat{b}}^{2-\hat{b}} \frac{1}{\left(\hat{v}_{t}+\frac{1}{R e_{\tau}}\right)} \mathrm{d} \hat{z} \\
& -\int_{\hat{b}}^{2-\hat{b}}\left[\frac{1}{\left(\hat{v}_{t}+\frac{1}{R e_{\tau}}\right)} \cdot\left(\int_{\hat{b}}^{\hat{z}^{\prime}} \hat{\mathrm{c}} \mathrm{d} \hat{z}^{\prime \prime}\right)\right] \mathrm{d} \hat{z}^{\prime},
\end{aligned}
$$

which, along with equations (37)-(39), can be solved iteratively for $\hat{u}_{*}$ and $\hat{u}_{*}$.

[37] The condition of zero net sediment flux is imposed at both boundaries:

$$
\begin{gathered}
\left.\hat{v}_{\mathrm{s} i} \hat{c}_{i}\right|_{\hat{z}=\hat{b}}=\left.\widehat{\widehat{w^{\prime} c^{\prime}}}\right|_{\hat{z}=\hat{b}}, \\
\left.\hat{v}_{\mathrm{si}} \hat{c}_{i}\right|_{\hat{z}=2-\hat{b}}=\left.\widehat{\left.\widehat{w^{\prime} c^{\prime}}\right|^{\prime}}\right|_{\hat{z}=2-\hat{b}}, i=1 \sim N .
\end{gathered}
$$

[38] These conditions alone, however, do not ensure a unique solution. The condition of conservation of total sediment concentration must also be implemented. This is given as

$$
\int_{\hat{b}}^{2-\hat{b}} \hat{c}_{i} \mathrm{~d} \hat{z}=p_{i}^{\mathrm{wc}}(2-2 \hat{b}), \quad i=1 \sim N
$$

where $p_{i}^{\mathrm{wc}}$ is the volumetric proportion of the sediment in the $i$ th fraction range in the water column. For flows containing single particle size, $p_{1}^{\mathrm{wc}}=1$.

[39] The boundary conditions for the equations governing the turbulence are obtained by assuming a near-wall balance between energy production and dissipation. These are given by

$$
\begin{aligned}
\left.\hat{q}^{3}\right|_{\hat{z}=\hat{b}} & =\left.\left(B_{1} \hat{l} \hat{v}_{t}\left(\frac{\partial \hat{u}}{\partial \hat{z}}\right)^{2}\right)\right|_{\hat{z}=\hat{b}} \\
& =B_{1} \kappa \hat{b}\left(\hat{u}_{*_{, b}}^{2}-\hat{b}-\frac{1}{R e_{\tau}}\left(\frac{\partial \hat{u}}{\partial \hat{z}}\right)_{\hat{z}=\hat{b}}\right)\left(\frac{\hat{u} *_{, b}}{\kappa \hat{b}}-\frac{\alpha \hat{B}_{b}}{\hat{u}_{*, b}^{2}}\right) ; \\
\left.\hat{q}^{3}\right|_{\hat{z}=2-\hat{b}} & =\left.\left(B_{1} \hat{l}_{\hat{v}_{t}}\left(\frac{\partial \hat{u}}{\partial \hat{z}}\right)^{2}\right)\right|_{\hat{z}=2-\hat{b}} \\
& =B_{1} \kappa \hat{b}\left(-\hat{u}_{*_{, t}}^{2}+\hat{b}-\frac{1}{R e_{\tau}}\left(\frac{\partial \hat{u}}{\partial \hat{z}}\right)_{\hat{z}=2-\hat{b}}\right)\left(-\frac{\hat{u} *_{, t}}{\kappa \hat{b}}+\frac{\alpha \hat{B}_{t}}{\hat{u}_{*, t}^{2}}\right) ;
\end{aligned}
$$

$$
\begin{aligned}
&\left.\hat{\varepsilon}\right|_{\hat{z}=\hat{b}}=\left.\left(\hat{v}_{t}\left(\frac{\partial \hat{u}}{\partial \hat{z}}\right)^{2}\right)\right|_{\hat{z}=\hat{b}}\left(\hat{u}_{*_{, b}}^{2}-\hat{b}-\frac{1}{R e_{\tau}}\left(\frac{\partial \hat{u}}{\partial \hat{z}}\right)_{\hat{z}=\hat{b}}\right)\left(\frac{\hat{u} *_{, b}}{\kappa \hat{b}}-\frac{\alpha \hat{B}_{b}}{\hat{u}_{*, b}^{2}}\right) ; \\
&\left.\hat{\varepsilon}\right|_{\hat{z}=2-\hat{b}}=\left.\left(\hat{v}_{t}\left(\frac{\partial \hat{u}}{\partial \hat{z}}\right)^{2}\right)\right|_{\hat{z}=2-\hat{b}}\left(-\hat{u}_{*, t}^{2}+\hat{b}-\frac{1}{R e_{\tau}}\left(\frac{\partial \hat{u}}{\partial \hat{z}}\right)_{\hat{z}=2-\hat{b}}\right)\left(-\frac{\hat{u} *_{, t}}{\kappa \hat{b}}+\frac{\alpha \hat{B}_{t}}{\hat{u}_{*, t}^{2}}\right) ; \\
&\left.\hat{k}^{2}\right|_{\hat{z}=\hat{b}}=\left.\left(\frac{\hat{v}_{t} \hat{\varepsilon}}{C_{\mu}}\right)\right|_{\hat{z}=\hat{b}}=\frac{1}{C_{\mu}}\left(\hat{u}_{*, b}^{2}-\hat{b}-\frac{1}{R e_{\tau}}\left(\frac{\partial \hat{u}}{\partial \hat{z}}\right)_{\hat{z}=\hat{b}}\right)^{2} ; \\
&\left.\hat{k}^{2}\right|_{\hat{z}=2-\hat{b}}=\left.\left(\frac{\hat{v}_{t} \hat{\varepsilon}}{C_{\mu}}\right)\right|_{\hat{z}=2-\hat{b}} \\
&=\frac{1}{C_{\mu}}\left(-\hat{u}_{*, t}^{2}+\hat{b}-\frac{1}{R e_{\tau}}\left(\frac{\partial \hat{u}}{\partial \hat{z}}\right)_{\hat{z}=2-\hat{b}}\right)^{2},
\end{aligned}
$$

where $\hat{B}_{b}=-R i_{\tau} \sum_{i} \hat{v}_{\mathrm{si}} \hat{c}_{\mathrm{bi}}$ and $\hat{B}_{t}=-R i_{\tau} \sum_{i} \hat{v}_{\mathrm{si}} \hat{c}_{\mathrm{ti}}$ represent buoyancy production at the bed and roof, respectively, and $\alpha$ is an empirical constant. In various geophysical flows as well as sediment-laden flows, it has been observed that stratification effects tend to increase the velocity gradient in the near-wall region [e.g., Monin and Obukhov, 1954; Turner, 1973; Einstein and Chien, 1955; Vanoni, 1946]. Here we include such effects in the boundary conditions by introducing a correction $\left(-\alpha \hat{B}_{b} / \hat{u}_{*, b}^{2}\right.$ at the bed and $\alpha \hat{B}_{t} / \hat{u}_{*, t}^{2}$ at the roof) to the velocity gradients 'under neutral conditions. This gives the velocity gradient in the form of $\hat{u}_{{ }_{,},} / \kappa \hat{b}-\alpha \hat{B}_{b} / \hat{u}_{*, b}^{2}$ at the bed and $\hat{u}_{*,} / \kappa \hat{b}-\alpha \hat{B}_{b} / \hat{u}_{*}^{2}$ at the roof [Turner, 1973; Yeh and Parker, 2013]. Note that in stably stratified flows, both $\hat{B}_{b}$ and $\hat{B}_{t}$ are negative. This corresponds to an increase in the magnitude of the velocity gradients at both boundaries.

[40] Finally, the boundary conditions for the master length scale are given by

$$
\left.\hat{l}\right|_{\hat{z}=\hat{b}}=\left.\hat{l}\right|_{\hat{z}=2-\hat{b}}=\kappa \hat{b}
$$

[41] The dimensionless governing equations and boundary conditions presented above indicate that the solutions are dependent on the set of dimensionless parameters $\left(R e_{\tau}, R i_{\tau}, \hat{v}_{s}, \hat{b}\right.$, $S c_{t}$, and $S c$ ). However, the reference height $\hat{b}$ only appears in the boundary conditions and does not have a strong influence 


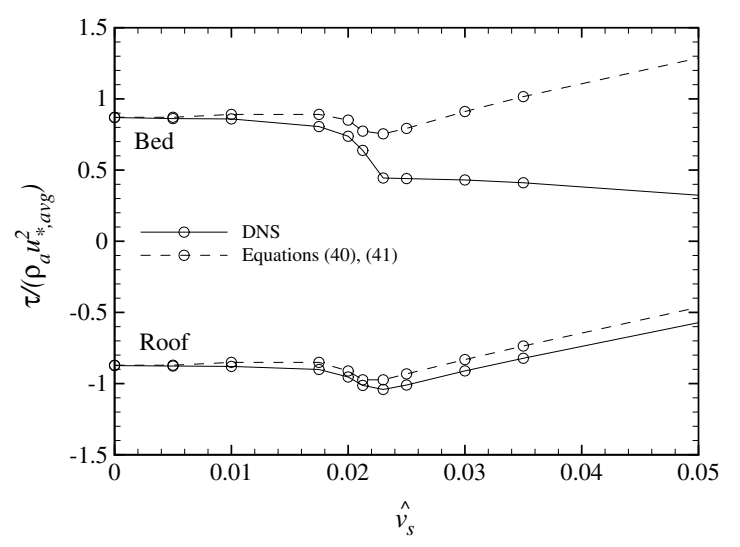

Figure 2. Comparison of dimensionless total roof and bed shear stresses as functions of dimensionless fall velocity at distance $\hat{b}$ away from the boundaries. Circles correspond to different simulation cases in $\mathrm{C} 09$ and this study.

on the stratification effects in the current body. The Schmidt number $S c$ and turbulent Schmidt number $S c_{t}$ denote the efficiency of the flow in mixing momentum versus sediment mass at a molecular and turbulent level, respectively. For sediment-laden flows, commonly used values for $S c_{t}$ range between 0.7 and 1.3 [Cellino and Graf, 1999; Bombardelli and Jha, 2009; Huang et al., 2004]. Here we set $S c_{t}=1$. The dependency of the solutions on $R e_{\tau}$ becomes rather weak when the value is sufficiently large. Such Reynolds invariance is similar to those found in pipe flows and open channels, and is likely true in sustained turbidity currents in nature.

[42] It can therefore be concluded that $\hat{v}_{s}$ and $R i_{\tau}$ are the dominant parameters in the present problem. The dimensionless settling velocity $\hat{v}_{s}$ is a function of, among other things the grain size, and thus encapsulates the tendency for the sediment to settle. Higher values of $\hat{v}_{s}$ create concentration profiles that are more biased toward the bed, so yielding higher stratification effects.

[43] Equations (8) and (22) can be used to show that the shear Richardson number $R i_{\tau}$ is equal to the inverse of slope:

$$
R i_{\tau}=\frac{1}{S_{0}} .
$$

[44] It can be seen, after some algebraic substitutions, that the appearance of $R i_{\tau}$ is always accompanied by the concentration gradient. For example, a reduction of (20) with (16) yields the result

$$
\hat{B}=\hat{v}_{t c} R i_{\tau} \frac{\partial \hat{c}}{\partial \hat{z}} .
$$

[45] In other words, in the absence of an internal density gradient (associated with, e.g., thermohaline effects or suspended sediment), $R i_{\tau}$ would exert no influence on density stratification regardless of its value. If the flow is stratified even just slightly, increasing values of $R i_{\tau}$ serve to magnify the importance of stratification effects up to several orders. In short, the role of $\hat{v}_{s}$ is to create a density gradient and associated stratification effects; increasing value of $R i_{\tau}$ acts to amplify them.

[46] It should be noted that the depth-averaged concentration $C$ in and of itself plays no role in the dimensionless solutions, but rather is absorbed into $R i_{\tau}$ according to equation (22). Instead, its influence is reflected in $u_{*}$,avg and is revealed only after conversion to dimensioned variables.

\section{Revisiting the DNS Simulations}

[47] Direct numerical simulations (DNS) were implemented by $\mathrm{C} 09$ to investigate stratification effects and the associated internal structure of turbidity currents under the setting of TCR. The value of $R e_{\tau}$ was set to 180 in all runs, a value chosen to reflect computational costs. Although this value appears relatively low in comparison to field or laboratory scales, C09 demonstrated that the resulting Reynolds number, defined as $R e=U h / v$ where $U$ is the depth-averaged velocity, reaches the low end of the Reynolds invariance in the case of unstratified flow. Based on this, they argued that their results for both unstratified and stratified flow can be extrapolated in more realistic scales. In both $\mathrm{C} 09$ and the present work, the value of $R i_{\tau}$ has been set equal set to 11.43 , which according to equation (53), corresponds to a $5^{\circ}$ slope. The effects of stratification on the mean flow and turbulent characteristics were then examined by varying $\hat{v}_{s}$ from 0 (neutral condition) to 0.05 (extreme stratification). The value of $\hat{v}_{s}$ for each case as well as some key results explained below are summarized in Table 3 .

[48] Several key findings were reported by C09. The flows modeled therein can be divided into two regimes. i.e., Regime I, for which $\hat{v}_{s}$ is less than about 0.022 (corresponding to cases 1-5 in Table 3), and Regime II, for which $\hat{v}_{s}$ is greater than about 0.022 (cases $6-10$ in Table 3). The value $\hat{v}_{s}=0.022$ corresponds to a threshold, beyond which turbulence in the lower half of the flow is not only damped but also nearly extinguished over a region. More specifically, within Regime II, turbulence is so strongly damped in the lower half that a zone with near-vanishing Reynolds stress and Reynolds flux appears. The only reason that sediment is maintained in suspension at all in this range is the effect of the artificial molecular diffusivity. This effect confines the suspended to a thin layer near the bed. Cantero et al. [2012] argued that this condition effectively corresponds to a turbidity current that has dropped its sediment out and died.

[49] As $\hat{v}_{s}$ increases from 0 up to this threshold value (Regime I, corresponding to cases 1-5), the sediment concentration profile gradually becomes biased toward the bed, as compared to the uniform profile prevailing for neutral conditions (i.e., case 0 , corresponding to a finite concentration of suspended sediment with vanishing fall velocity). The uneven driving force throughout the water column results in an asymmetry of the velocity profile relative to the centerline, with point of maximum velocity $\hat{z}_{u, \max }$ biased toward the bed. The velocity profile below the maximum can be fitted to a logarithmic law, but the effect of stratification is reflected in a reduced value of $\kappa$ computed from the velocity gradient. The RMS of the velocity fluctuations, which indicates the level of turbulence, decreases with increasing $\hat{v}_{s}$ within the lower half of the channel, due to damping of turbulence caused by stratification effects. Within the upper part of the channel, however, the turbulent kinetic energy increases due to the deficit in both sediment concentration and concentration relative to the lower part of the channel. This deficit results in less stratification effects. Within this regime, however, turbulence is maintained throughout the water column.

[50] The flow changes dramatically as $\hat{v}_{s}$ increases into the range of Regime II (cases 6-10). In this regime, the above tendencies for the mean flow characteristics are further 
Table 3. Settings and Partial Results From Cantero et al. [2009]

\begin{tabular}{lcccc}
\hline Case & $\hat{v}_{s}$ & $\hat{c}_{b}{ }^{\mathrm{a}}$ & $\hat{z}_{u, \max }{ }^{\mathrm{b}}$ & $C_{f}{ }^{\mathrm{c}}$ \\
\hline 0 & 0 & 1 & 1 & 0.0085 \\
1 & 0.005 & 1.06 & 0.97 & 0.0082 \\
2 & 0.010 & 1.16 & 0.93 & 0.0079 \\
3 & 0.0175 & 1.44 & 0.8 & 0.0074 \\
4 & 0.02 & 1.59 & 0.74 & 0.0069 \\
5 & 0.02125 & 1.71 & 0.71 & 0.0065 \\
6 & 0.023 & 2.59 & 0.42 & 0.0061 \\
7 & 0.025 & 2.75 & 0.42 & 0.0063 \\
8 & 0.03 & 3.22 & 0.37 & 0.0068 \\
9 & 0.035 & 3.61 & 0.32 & 0.0074 \\
10 & 0.05 & 4.38 & 0.25 & 0.0102 \\
\hline
\end{tabular}

$\mathrm{a}_{\hat{c}_{b}}$, dimensionless near-bed (at $\hat{z}=\hat{b}$ ) concentration, equivalent to $r_{0}$.

$\mathrm{b}_{\hat{z}_{u, \text { max}}}$, location of velocity maximum.

${ }^{\mathrm{c}} C_{f}$, flow resistance coefficient.

enhanced, but even an adjusted version of the conventional logarithmic law is no longer capable of describing the nearbed velocity distribution. Moreover, a significant and sudden drop in turbulent kinetic energy is observed in a zone near the bed as the threshold is crossed. This phenomenon, which may be referred to as flow relaminarization, is reflected in the negligible contribution of the Reynolds stress to the total shear stress and the Reynolds flux of suspended sediment to the total flux. The extent of the region of nearly extinct is found to increase with increasing $\hat{v}_{s}$. This sudden collapse of turbulence as the threshold in $\hat{v}_{s}$ is crossed has important implications for sedimentology. Once the near-bed turbulence has collapsed, no mechanism is available to resuspend sediment when it settles on the bed. The result would be the gradual extinction of the current itself, along with the emplacement of a "massive," i.e., structureless deposit that is not reworked by bed load [Cantero et al., 2012].

[51] The results from C09 provide a basis for validating and understanding the limits of RANS models in predicting flow stratification effects and the internal structure of turbidity currents. The comparisons in the next few sections follow closely their study cases. In order to ensure the comparability of the model results to $\mathrm{C} 09$, it is useful to clarify here some of the differences between the two approaches.

[52] First of all, the governing equations presented in this study are strictly 1-D. They describe the vertical structure of the flow by assuming flow uniformity in the streamwise and cross-channel directions. In $\mathrm{C} 09$, such uniformity is achieved by setting a finite simulation domain and implementing periodic boundary conditions in both directions. Therefore, although the dimensions involved in the governing equations and the simulation domain differ between the two approaches, the flow characteristics which the two systems aim to capture are identical and the results are directly comparable.

[53] In principle, turbulence modeling using RANS models is only applicable under fully turbulent flow conditions. In particular, in the near-wall region where the viscous effects cannot be neglected, special treatment is required. Such treatment includes applying a wall function to describe the near-wall behavior or switching to a low Reynolds number model where the flow in the near-wall region is resolved [see e.g., Patel et al., 1985]. The flow settings examined in $\mathrm{C} 09$ is in the lower end of the Reynolds invariant region, and thus, discrepancy in the RANS and DNS results can be expected in the near-wall region. However, instead of applying a low Reynolds number treatment, calculation within this region is avoided by assigning a reference height (see section 2.3) and appropriate boundary conditions. Although such treatment may seem less accurate, it has several practical values. First of all, in simulations which are coupled with mass conservation of the bed, the sediment entrainment rate is typically referenced to a distance from the bed, due to difficulties in instrumentation [Garcia and Parker, 1991]. Hence, the flow field below that reference height is irrelevant as long as the boundary conditions at the reference height are appropriately specified. In addition, resolving flow into the viscous sublayer requires very fine gridding, which makes the approach computationally inefficient when applied at a field scale.

\section{Numerical Method and Validation}

[54] The vertical profiles are obtained by solving equations (11), (12), (17), (18), (28), and (29) numerically until convergence to steady state is attained. Discretization is done using a fully implicit finite volume method with a second-order accurate total variation diminishing scheme [Versteeg and Malalasekera, 2007]. Yeh and Parker [2013] have tested this scheme in an investigation of stratification effects due to suspended sediment in open channel flows. It is shown there that in order to capture large gradients in the profiles as a result of strong stratification effects, small grids are required. In other words, the accuracy of the model depends on both the level of stratification and the dimensionless grid size $d \hat{z}$. With this in mind, a fine grid of 801 points uniformly distributed over the computational domain is used in all simulations in this study. We use the time interval $d \hat{t}=0.01$.

[55] The model performance is tested with two limiting cases: a fully turbulent flow under neutral conditions and a fully "relaminarized" flow in the presence of strong stratification. The latter case may seem somewhat idealized for selfstratified flows, since the density gradient far away from the bed is almost always too small to create stratification effects strong enough to relaminarize the flow therein. Such strong stratification effects throughout the whole water column can be achieved, however, in the case of flows with externally forced density gradients (e.g., imposed temperature gradient over a Poiseulle flow). Here this case is tested for the sake of model validation.

[56] The comparison for the neutral case (case 0 ) is shown in Figure 3. It can be seen that all the RANS models predict velocity profiles which agree well with DNS results. The turbulent kinetic energy (TKE) profiles predicted by RANS are overall in satisfactory agreement with DNS but show deviation from DNS near the boundaries due to viscous effects. For fully relaminarized flows, the model results are compared with analytical solutions (see Appendix A). In the simulations, fully relaminarized conditions are achieved by setting the eddy viscosity to zero throughout the run. Figure 4 shows that the RANS models with turbulence turned off reproduce precisely the analytical solutions in the absence of turbulence. While the outcome of this numerical experiment may seem obvious, it demonstrates the potential of the present models to deal with possible spatial transition from a turbulent regime to a fully laminar regime in the presence of strong stratification. 

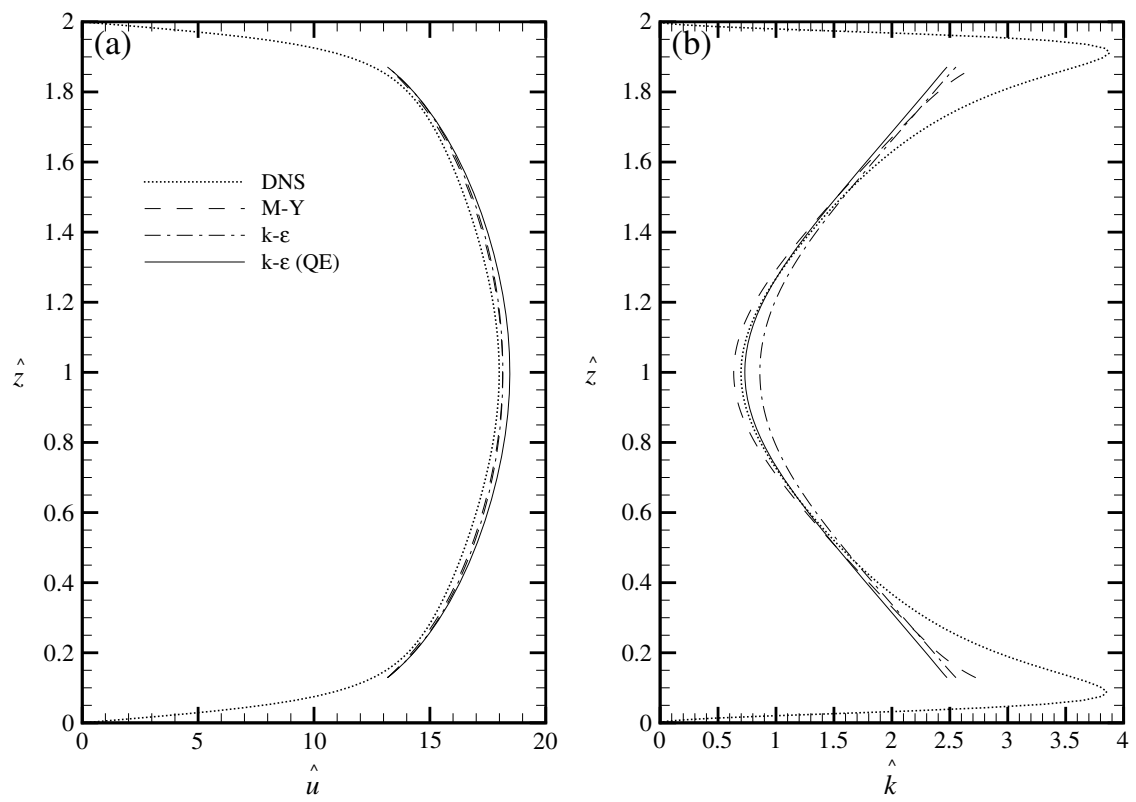

Figure 3. Comparison of the model results versus DNS for neutrally stratified flow. (a) velocity profile; (b) TKE profile.

\section{Regime I Stratification}

[57] The variation in the flow velocity and sediment concentration for cases 1, 3, and 5 are shown in Figures 5 and 6, respectively. The previously described flow characteristics are clearly seen in the DNS solutions. With increasing settling velocity, the sediment concentration profile becomes more biased toward the bed, with an increase in the near-bed concentration $\hat{c}_{b}$ from unity to approximately 1.7 for case 5 . The shift of driving force toward the lower part of the channel is also reflected in the increasing asymmetry exhibited by the velocity profiles. Based on the DNS results, the location for the velocity maximum is found to drop below the channel centerline to $\hat{z}_{u, \max }=0.71$ for case 5 .

[58] For weakly stratified flows, the mean flow characteristics predicted by DNS are captured reasonably well by all three RANS models. The difference between the standard and QE k- $\varepsilon$ models at this level of stratification is mainly due to the choice of the parameters $C_{\varepsilon 3}$ and $\sigma_{\varepsilon}$, since the density gradient is small enough so that $c_{\mu} \approx c_{\mu}^{0}$. However, as stratification effects increase, the choice of the stability
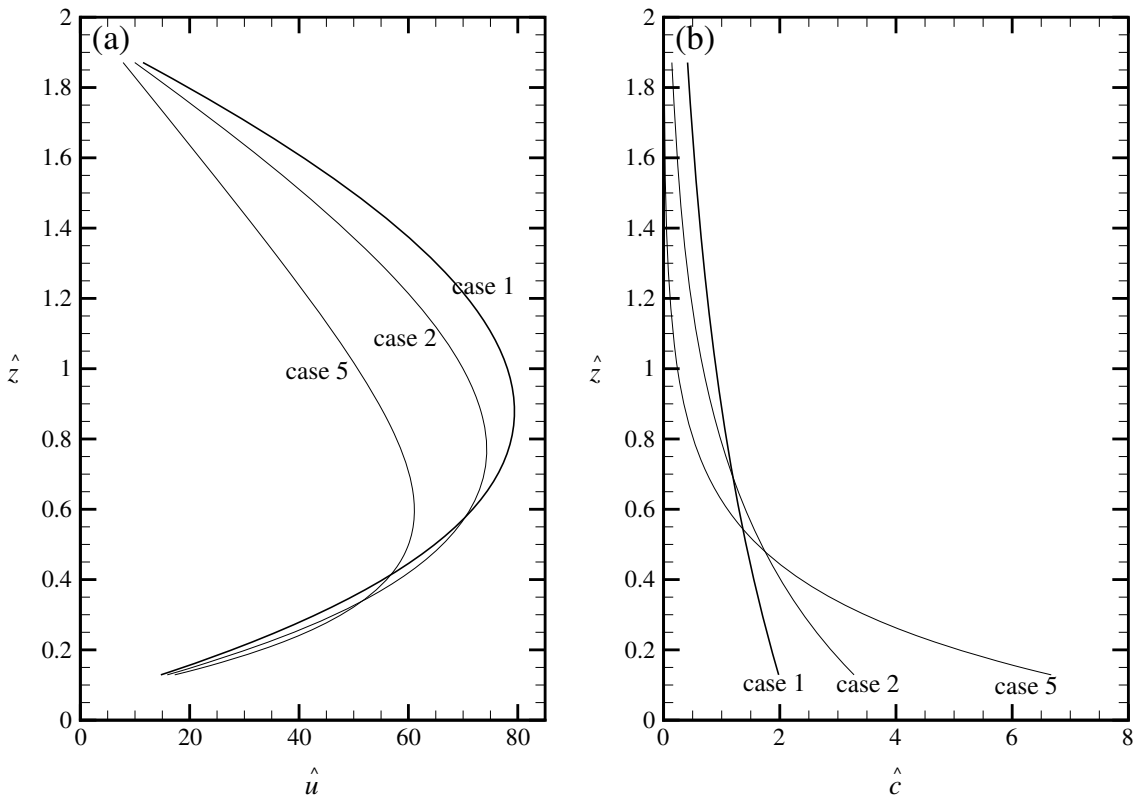

Figure 4. Comparison of the model results versus analytical solutions for fully relaminarized flow. (a) velocity profile; (b) concentration profile. All lines collapse onto the analytical solutions, indicating that the models replicate the laminar solutions when the flow fully relaminarizes. 

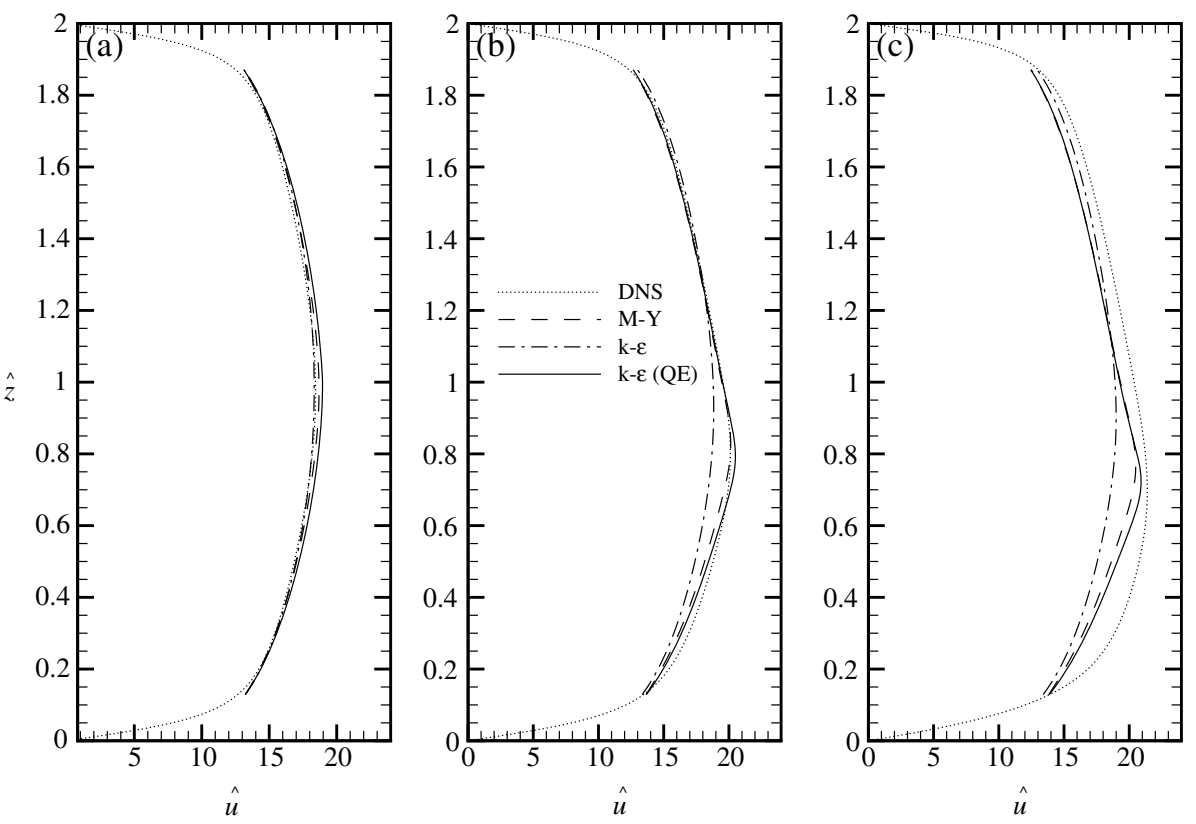

Figure 5. Comparison of Regime I velocity profiles predicted by DNS against the RANS results predicted

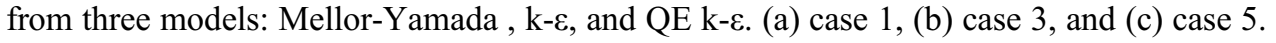

function becomes significant. In case 3, both $\mathrm{M}-\mathrm{Y}$ and $\mathrm{QE}$ $\mathrm{k}-\varepsilon$ capture the magnitude as well as the location of the velocity maximum. On the other hand, the standard $\mathrm{k}-\varepsilon$ model fails to accurately capture the level of stratification. Such a lukewarm performance extends through Case 5 where the model predicts a drop only to $\hat{z}_{u, \max }=0.9$ as opposed a drop to approximately $\hat{z}_{u, \max }=0.75$ predicted by both $\mathrm{M}-\mathrm{Y}$ and QE k- $\varepsilon$ (Figure 5c).

[59] Similar results are found in the concentration profiles. The standard k- $\varepsilon$ model tends to overestimate the eddy diffusivity and hence the degree of homogenization of sediment concentration in the flow. As shown in Figure 6, this results in underestimation of the near-bed concentration. It should be noted that while $\mathrm{M}-\mathrm{Y}$ and QE $\mathrm{k}-\varepsilon$ give predictions of near-bed concentration the more closely reflect DNS, the concentration profiles are not particularly well represented by any of the RANS models. This is especially true under strongly stratified conditions.

[60] Under such conditions, the RANS models tend to produce two distinct concentration layers separated by a large-density gradient (although this is not apparent for the standard $\mathrm{k}-\varepsilon$ model due to the relatively weak stratification effects). Unlike the formation of a lutocline in oceanic or limnological flows, this results from underestimation of the
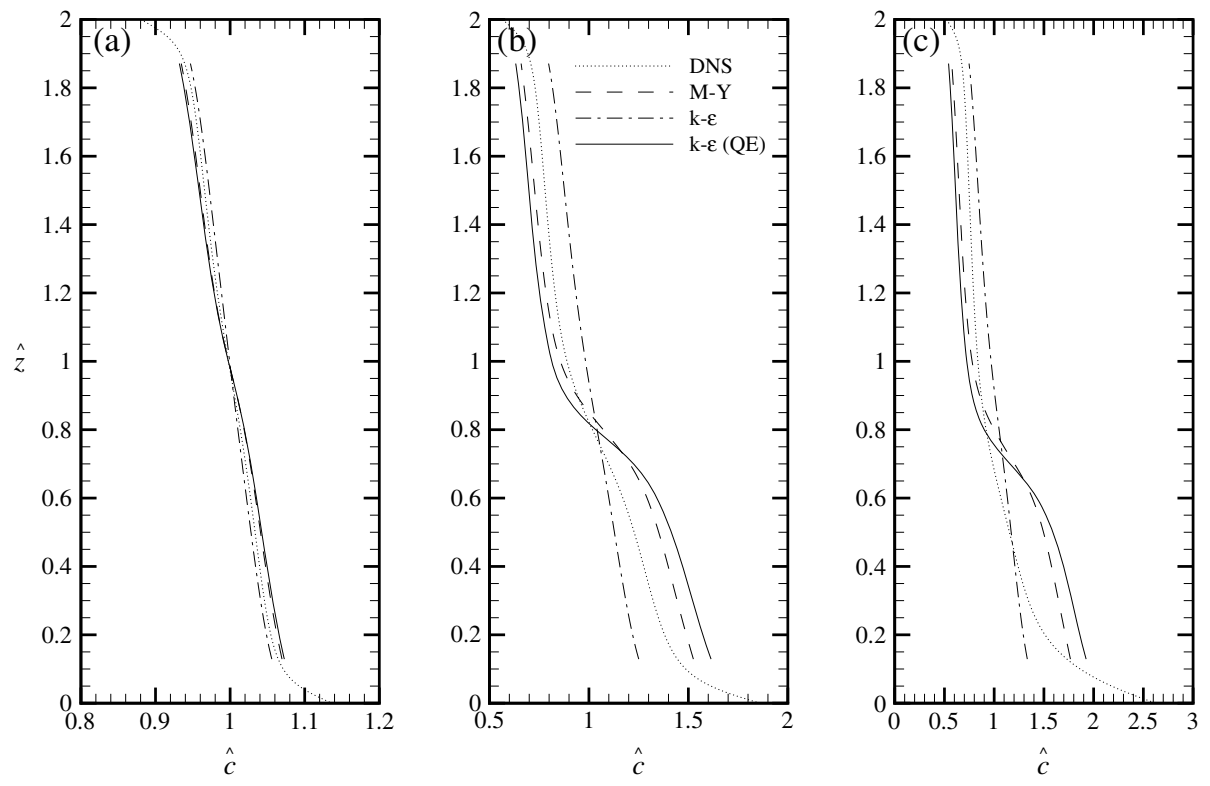

Figure 6. Comparison of Regime I concentration profiles predicted by DNS against the RANS results. (a) case 1 , (b) case 3, and (c) case 5 . 

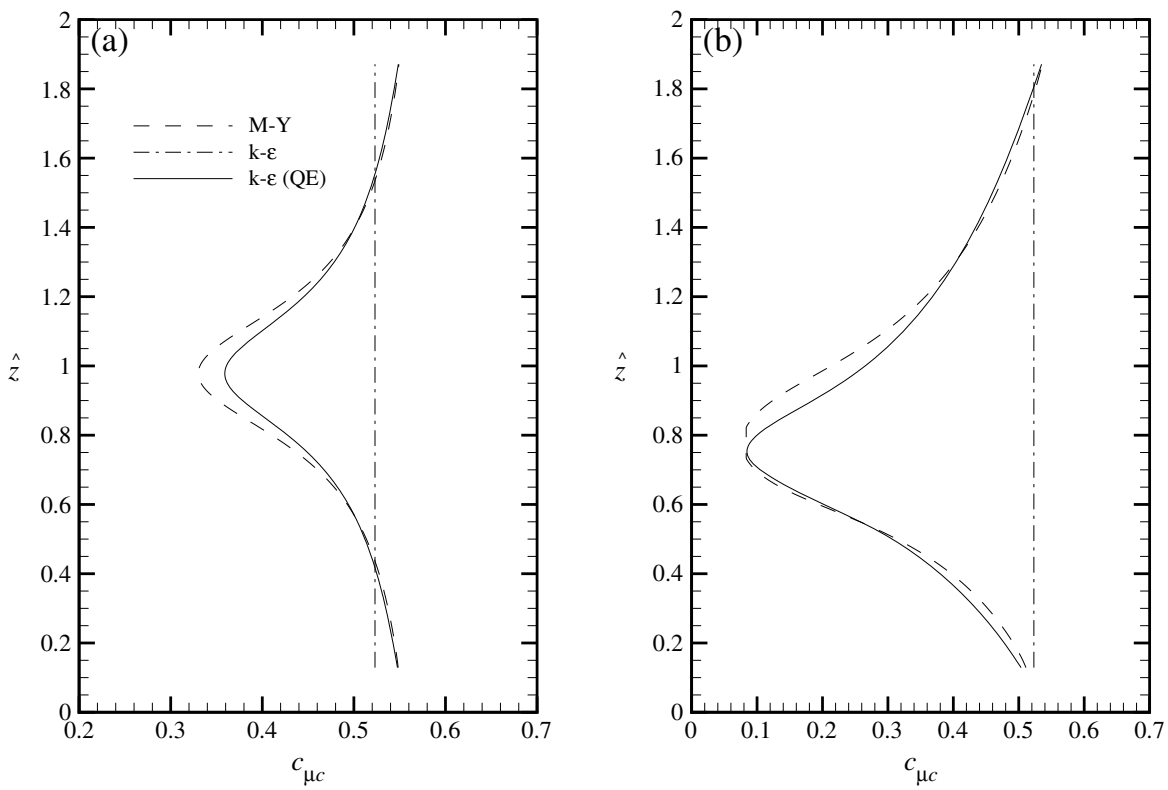

Figure 7. Comparison of $c_{\mu c}$ profiles for (a) case 1 and (b) case 5 . Higher damping is found in the vicinity of velocity maximum for M-Y and QE k- $\varepsilon$. The value of $c_{\mu c}$ for the standard $\mathrm{k}-\varepsilon$ model is calculated using equation (36).

Reynolds stress and flux by the RANS models in the vicinity of the velocity maximum. This phenomenon, which may be termed the "fish trap effect," inhibits the upward mixing of sediment from the near-bed region. Due to the form of the closure relations (14) and (15), the location for zero Reynolds stress and flux inevitably coincides with that of velocity maximum. This is inconsistent with the findings from C09, in which it is shown (Figure 16a therein) that such a coincidence occurs only under neutral conditions. As the flow becomes stratified, the locations of zero Reynolds stress and zero velocity gradient become displaced. This indicates that both mass and momentum are still being mixed by turbulence at the velocity maximum. The RANS turbulence closures used here completely shut off such processes. Such an effect is further accentuated by the use of stability functions in the M-Y and QE k- $\varepsilon$ models. These stability functions, as given by equations (25) and (26), describe the secondary damping of turbulence, where the level of damping increases with decreasing TKE. Figure 7 illustrates the variations in the stability function $c_{\mu c}$. For $\mathrm{M}-\mathrm{Y}$ and $\mathrm{QE} \mathrm{k}-\varepsilon$, the distribution of $c_{\mu c}$ is nonuniform, with the low values found in the vicinity of low TKE. The minimum of $c_{\mu c}$ is reduced as the flow becomes more stratified (Figure $7 \mathrm{~b}$ ). Such bias in the $c_{\mu c}$ profile places an emphasized damping on the eddy diffusivity in the low-TKE region and therefore accentuates the artificial lutocline.

[61] Comparisons of the TKE and eddy viscosity profiles for cases 1 and 5 are shown in Figures 8 and 9, respectively. The DNS estimate of the eddy viscosity is computed from equations (14) and (15) as $\hat{v}_{t}=-\widehat{\widehat{u^{\prime} w^{\prime}}} /(\partial \hat{u} / \partial \hat{z})$, a definition which perforce results in discontinuity at the velocity maximum. It can be seen that all RANS model results are generally in good agreement with those from DNS when stratification effects are weak. Close to the boundary, deviation from DNS is observed due to the choice of $\hat{b}$, as discussed in the previous section. In addition, the standard $k-\varepsilon$ model tends to overestimate TKE relative to DNS in the midchannel region, so resulting in an eddy viscosity profile that significantly differs from the others. Such results can be improved by fine-tuning the coefficients. However, as shown in the comparison for the mean flow profiles, these errors in the turbulence characteristics do not significantly affect the predictions in the flow velocity or sediment concentration, since the deviation from neutral conditions is rather weak. At case 5, just before the flow transition to Regime II, all RANS models tend to underestimate TKE and the eddy viscosity above the discontinuity. Below the discontinuity, it is intriguing to find that the RANS models predict lower TKE but higher eddy viscosity compared to DNS. This suggests that for the same energy level, energy is more efficiently dissipated when the local density gradient is large. These turbulence closures may thus become less suitable in describing the energy balance therein under such conditions.

[62] To quantify the level of stratification represented by the models, two dimensionless parameters are introduced: the gradient Richardson number and the damping efficiency. The gradient Richardson number is defined as

$$
R i_{g}=-R i_{\tau} \frac{\frac{\partial \hat{c}}{\partial \hat{z}}}{\left(\frac{\partial \hat{u}}{\partial \hat{z}}\right)^{2}}
$$

[63] This parameter can be viewed as an alternative form for the related flux Richardson number

$$
R i_{f}=-\frac{R i_{\tau} \overline{\dot{w}^{\prime} c^{\prime}}}{\overline{\dot{u}^{\prime} w^{\prime}}\left(\frac{\partial \hat{u}}{\partial \hat{z}}\right)},
$$

which represents a ratio between the buoyancy and energy production. In the RANS modeling presented here, equations (14) and (15) and the assumption $S c_{t}=1$ ensure that the two are equal to each other. 

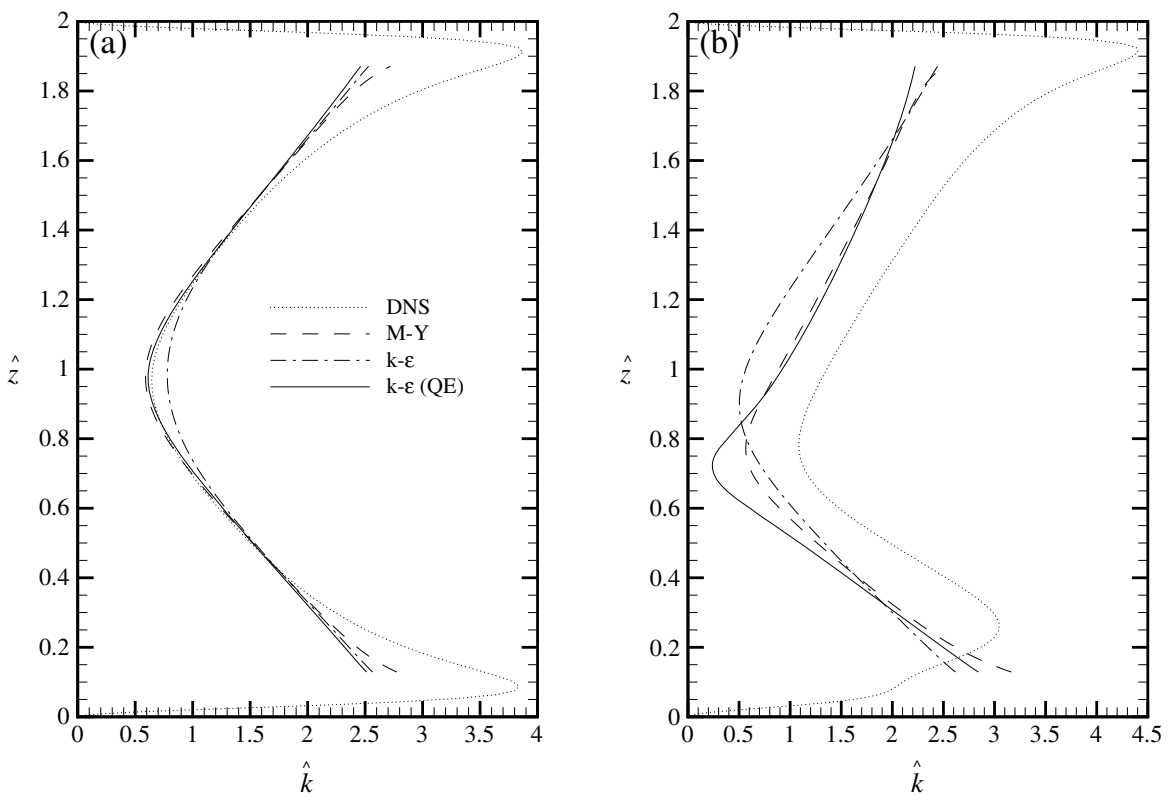

Figure 8. Comparison of Regime I TKE profiles predicted by DNS against the RANS results. (a) case 1 and (b) case 5.

[64] The damping efficiency is defined as DE $=\hat{v}_{t} / \hat{v}_{t 0}$. The relation between $R i_{g}$ and $\mathrm{DE}$ near the lower boundary is shown in Figure 10. In case 1, the damping efficiency predicted by the $\mathrm{M}-\mathrm{Y}$ and modified $\mathrm{k}-\varepsilon$ models resemble that from DNS up to $\hat{z}=0.5$. Toward the velocity maximum, $R i_{g}$ rapidly increases toward infinity and significant deviation of the damping efficiency from DNS can be expected. In the strongly stratified condition of case 5, however, these models are able to capture the damping effect only above $\hat{z}=0.5$. As seen in Figure $9 b$, substantial error relative to DNS is observed below $\hat{z}=0.5$ due to the failure of these models to account for the loss of energy under strongly stratified conditions. One may argue that such discrepancy originates from inappropriate boundary conditions specified for the RANS models. Further numerical tests showed, however, that forcing the boundary conditions used in RANS to match the DNS results does not improve the discrepancy. Indeed, the RANS predictions resulting from this forcing were found to be clearly erroneous, in that the sediment concentration is substantially overestimated in the lower part of the channel and TKE is substantially underestimated in the upper part of the midchannel. It is hence concluded that the turbulence
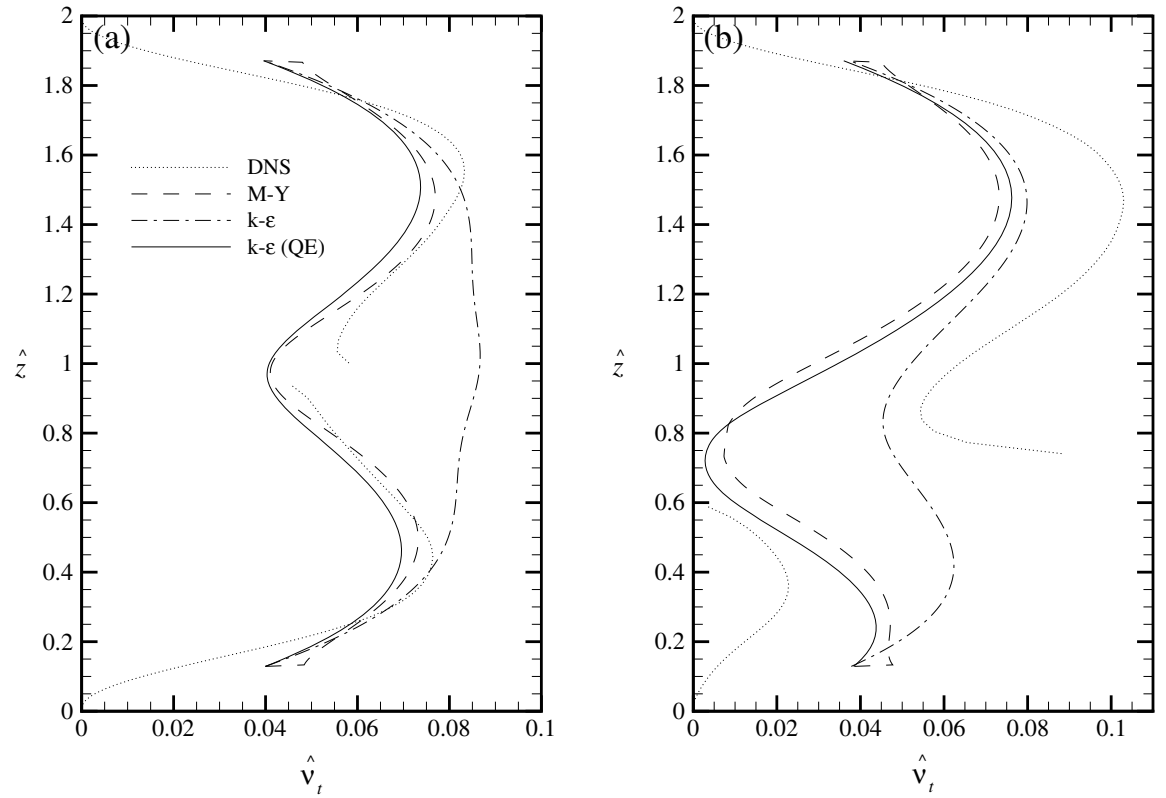

Figure 9. Comparison of Regime I eddy viscosity profiles predicted by DNS against the RANS results. (a) case 1 and (b) case 5 . DNS estimate of the eddy viscosity is computed by $\hat{v}_{t}=-\overline{\overline{u^{\prime} w^{\prime}}} /(\partial \hat{u} / \partial \hat{z})$. 

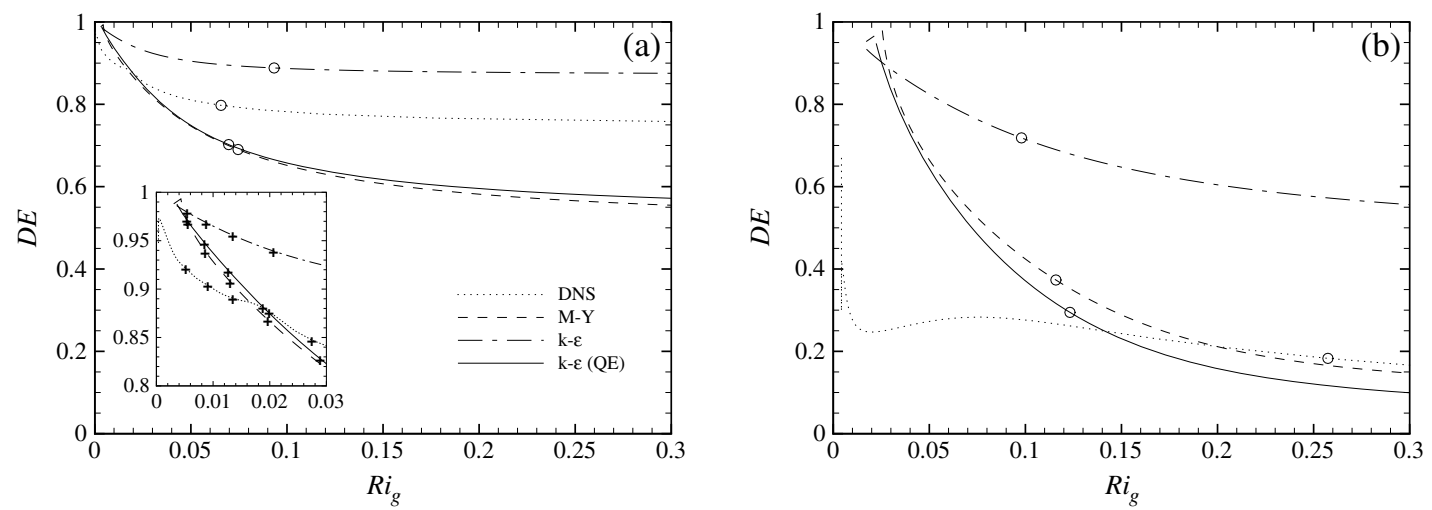

Figure 10. Model comparisons for damping efficiency as a function of gradient Richardson number. Increasing value of $R i_{g}$ along a line corresponds to increasing $\hat{z}$, i.e., farther away from the bed. (a) Case 1 ; circles indicate the values at $\hat{z}=0.75$. The inset shows the values in the near-bed region. The leftmost cross on each line indicates the value at $\hat{z}=0.2$. Every cross marks an increment of $d \hat{z}=0.1$. (b) Case 5 ; circles indicate the values at $\hat{z}=0.5$.

closures themselves, rather than the boundary conditions, are responsible for failure of the RANS models to reproduce the DNS results under strongly stratified conditions.

\section{Regime II Stratification}

[65] With further increase in $\hat{v}_{s}$, the flow enters Regime II. Figures 11 and 12 show a comparison of the DNS and RANS results for mean flow and turbulent characteristics associated with cases 6 and 10, respectively. In Regime II, the flow obtained from DNS is characterized by near- or complete local relaminarization near the bed, where the TKE is highly (or sometimes completely) damped (compare Figures $8 \mathrm{~b}$, $11 \mathrm{c}$, and $12 \mathrm{c}$ ). In addition, the local TKE maximum in the lower part of the channel characteristic of Regime I (seen in Figure $8 \mathrm{~b}$ ) vanishes (Figures $11 \mathrm{c}$ and $12 \mathrm{c}$ ). As the flow is no longer able to suspend sediment by turbulence, suspended sediment collapses to a thin near-bed layer, where suspension is maintained by the effect of the artificial molecular diffusivity. A highly skewed, nose-like velocity profile with $\hat{z}_{u \text {, max }}$ very close to the bed is thus created (Figure 12a). Comparisons between case 5 (highest value of $\hat{v}_{s}$ within Regime I) and case 6 (lowest value of $\hat{v}_{s}$ within Regime II) show that results from all RANS models reflect only the slight change in $\hat{v}_{S}$ (from 0.02125 in case 5 to 0.23 in case 6 ), and fail to reflect the transition between two different flow regimes. The velocity profiles show that a weak drop of $0.02-0.03$ in $\hat{z}_{u, \max }$ from $\hat{v}_{s}=0.02125$ to $\hat{v}_{s}=0.023$ is predicted using the M-Y and QE k- $\varepsilon$ models, whereas DNS predicts a drop that is approximately 10 times larger. The RANS models also fail to reproduce the damping of TKE below the velocity maximum. As $\hat{v}_{s}$ increases, the discrepancy of the mean flow and turbulent characteristics between the RANS models and DNS is further enhanced (Figure 12). It should be kept in
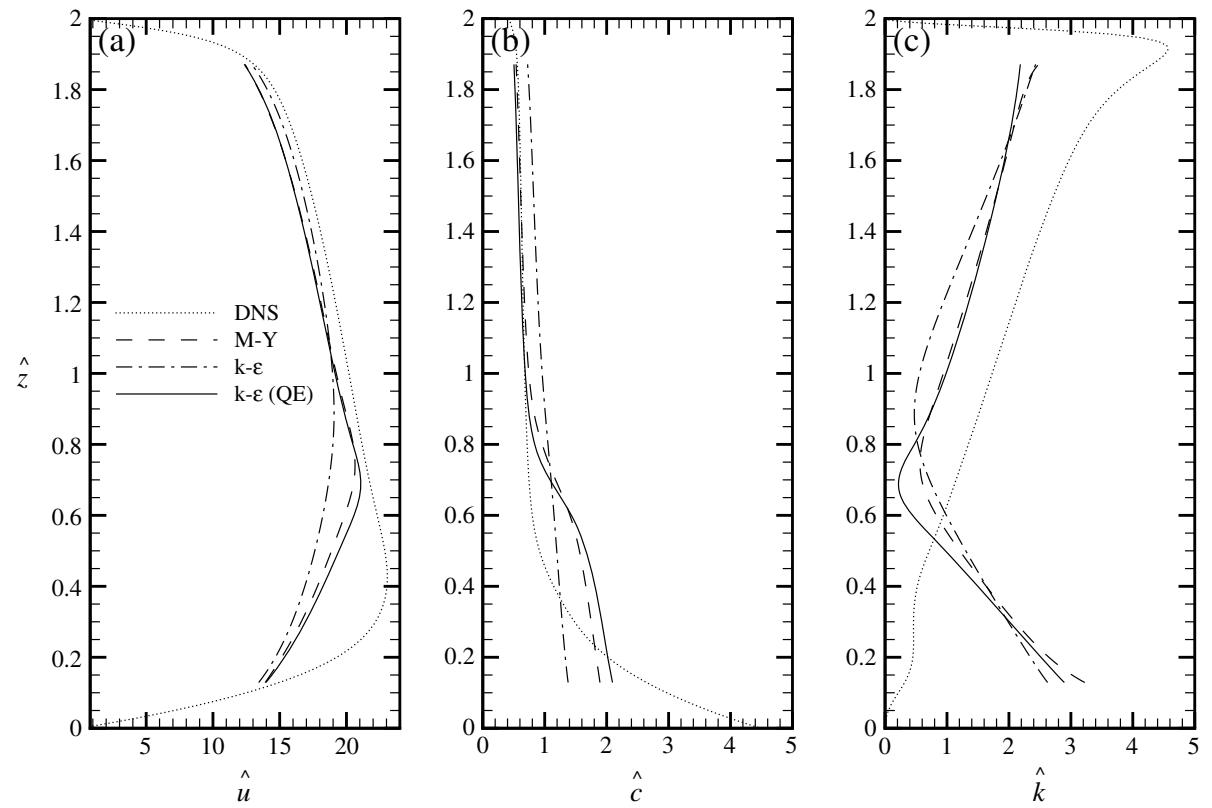

Figure 11. Comparison of (a) velocity profile, (b) concentration profile, and (c) TKE profile for case 6. 

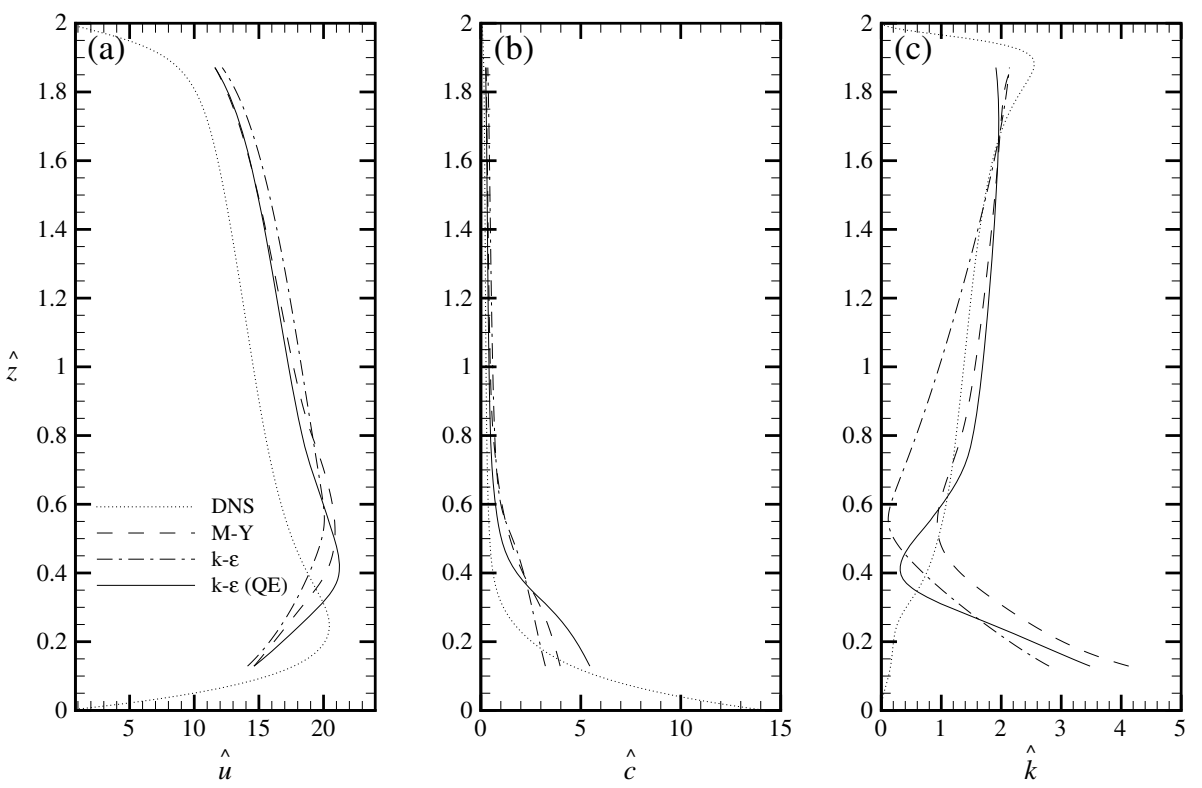

Figure 12. Comparison of (a) velocity profile, (b) concentration profile, and (c) TKE profile for case 10.

mind, however, that it was shown in Figure 2 that the boundary conditions are partially responsible for failure in this case.

[66] The ratio between the Reynolds flux $F_{R e}=-\overline{w^{\prime} c^{\prime}}$ and viscous flux $F_{v}=v_{c} \mathrm{~d} c / \mathrm{d} z$ of suspended sediment are shown in Figure 13 for cases 5 and 6 . The DNS results show that just before relaminarization occurs, the near-bed sediment flux due to turbulence, although highly damped, maintains values that are larger than that associated with the artificial molecular viscosity. When the flow relaminarizes, the dominance of $F_{v}$ is seen for values of $\hat{z}$ up to 0.4 , indicating the sediment in that region is no longer suspended by turbulence.

[67] We emphasize that the molecular flux is indeed artificial. The appropriate interpretation of the DNS results is that for a given value of $R i_{\tau}$, sufficiently large values of $\hat{v}_{S}$ result in such strong stratification that the near-bed turbulence is

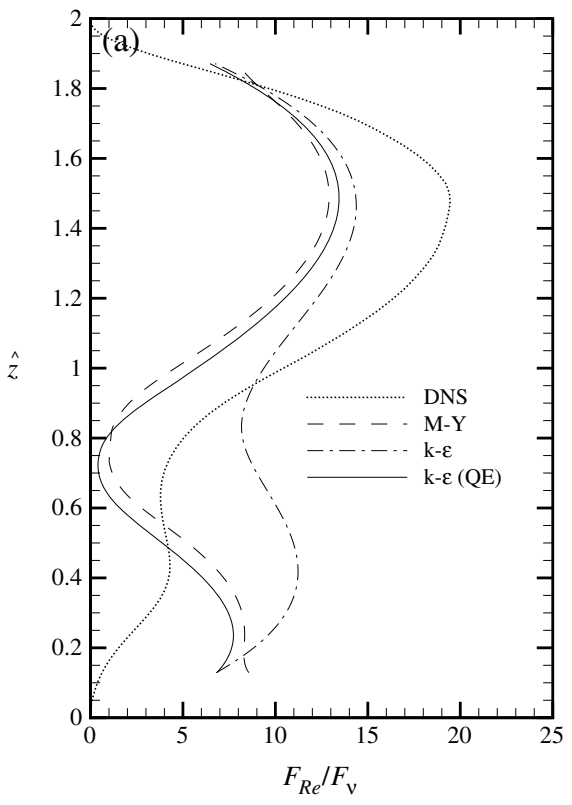

Figure 13. Model comparisons for $F_{R e} / F_{v}$ profiles. (a) Case 5 (Regime I) and (b) case 6 (Regime II). extinguished. In real flows, sediment would settle out not into the thin layer dictated by the artificial molecular viscosity, but instead to the bed itself, never to be reentrained. That is, within Regime II, a self-sustaining turbidity current is no longer possible [Cantero et al., 2012]. Figure 14, which shows the ratio between the Reynolds stress $\tau_{R e}=-\rho_{a} \overline{u^{\prime} w^{\prime}}$ and the viscous stress $\tau_{v}=\rho_{a} v \partial u / \partial z$, illustrates the region over which such a phenomenon occurs. As the flow transitions into Regime II, the momentum flux is dominated by the artificial molecular viscosity below $\hat{z} \approx 0.35$ in case 6 . The extent of such a region is found in C09 to increase with the level of stratification (increasing $\hat{v}_{S}$ in the present case). It is clear from Figures 11c, 12c, 13b, and 14b that the RANS models are unable to capture the phenomenon of collapse of near-bed turbulence under conditions of strong self-stratification. As a

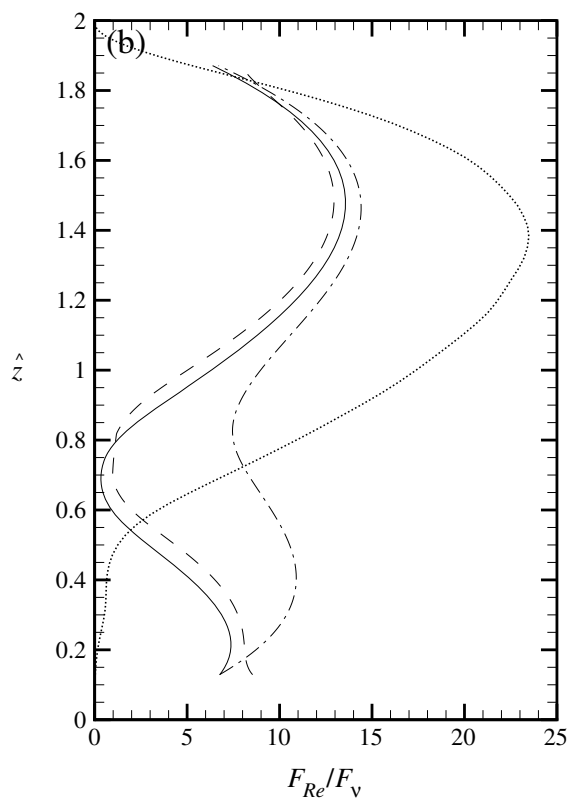




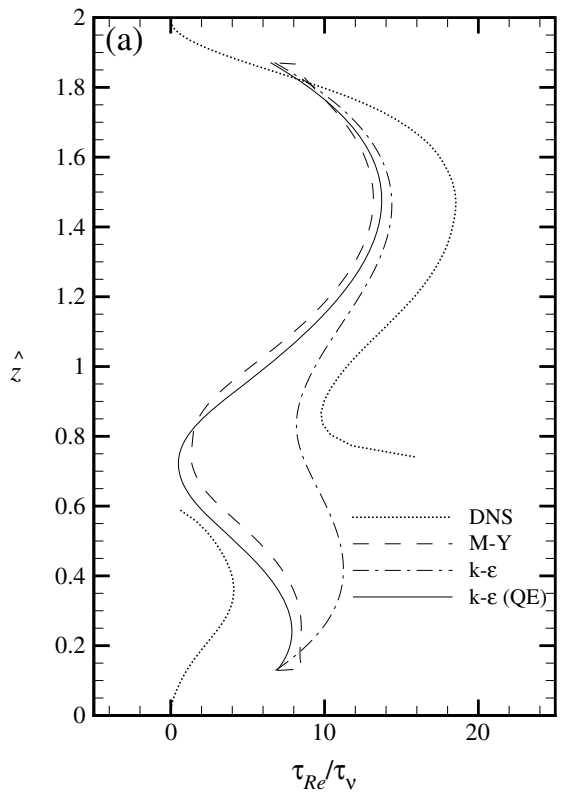

Figure 14. Model comparisons for $\tau_{R e} / \tau_{v}$ profiles. (a) Case 5 (Regime I) and (b) case 6 (Regime II).

result, they fail to capture a phenomenon that should result in (a) rainout of suspended sediment from the current without reworking or resuspension, (b) emplacement of a structureless (massive) deposit, and (c) eventual extinction of the turbidity current itself.

[68] A perusal of Figures 5, 6, 8, 9, 13, and 14 reveals that even before the flow makes the transition to Regime II, the RANS turbulence closures used here fail to fully describe the turbulence field under strongly stratified conditions. This failure is likely due to their inability to capture the transition of energy from turbulence to internal waves, a phenomenon which has been observed in various geophysical flows [Müller et al., 1986; Gregg, 1987, 1989]. This is illustrated in Figure 15. The right-hand side column of Figure 15 shows the production $(\hat{P})$ and dissipation $(\hat{\varepsilon})$ rates of TKE predicted by DNS for cases 5 and 6. It is apparent that as the flow relaminarizes, the magnitude of both rates are significantly reduced. Note that the depth-average velocity $U$ reported in C09 for cases $5(=17.48)$ and $6(=18.04)$ are of the same order. This implies that the energy is likely to be more efficiently transferred to and dissipated through internal waves. The RANS models used in this study, which do not describe the additional energy sinks, clearly fail to capture the reductions in $\hat{P}$ and $\hat{\varepsilon}$ (Figures 15 a and 15c).

[69] More evidence of energy dissipation through internal waves can be seen in the variation of the characteristic length scales. Under non-stratified conditions, all energycontaining eddies eventually lose their energy to viscous dissipation at a length scale characterized by the Kolmogorov scale $l_{k}=\left(v^{3} / \varepsilon\right)^{1 / 4}$. However, in the presence of density stratification, the movement of the fluid particles is limited not only by the largest scales possible (e.g., flow scale) but also by the available energy to mix the excess density against the density gradient. In other words, the maximum excursion of a fluid particle is set by converting all its (vertical) TKE to potential energy. This length scale is characterized by the buoyancy scale $l_{b}=\left(\overline{w^{\prime 2}}\right)^{1 / 2} / N_{\mathrm{BV}}$

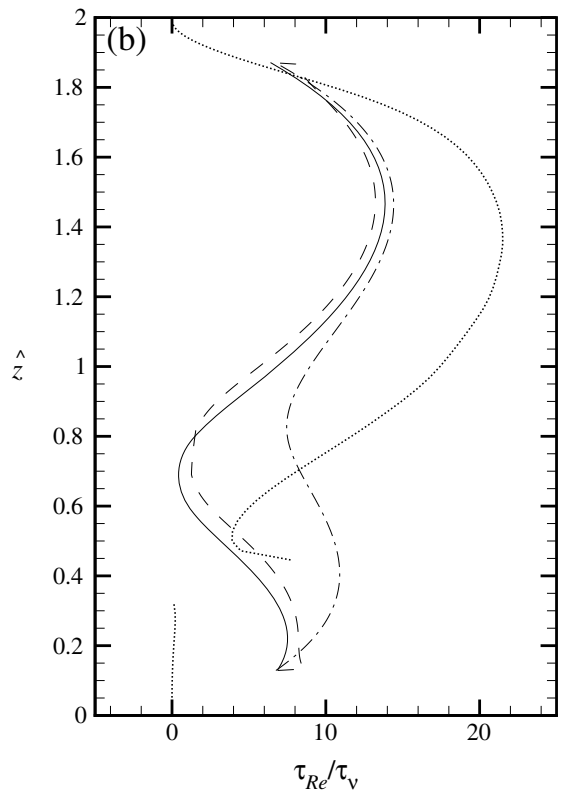

where $N_{\mathrm{BV}}=(-\operatorname{Rg}(\partial c / \partial z))^{1 / 2}$ is the Brunt-Väisälä frequency. The scaling $l \sim q^{3} / \varepsilon$ (equation (34)) allows the buoyancy length scale to be expressed alternatively in terms of the Ozmidov scale $l_{o}=\left(\varepsilon / N_{\mathrm{BV}}^{3}\right)^{1 / 2}$.

[70] For turbulent length scales below this threshold, energy is dissipated in a fashion similar to a non-stratified flow. With the increase in the level of stratification, the larger scales are suppressed by the buoyancy force, whereas the scales still below the buoyancy scale limit follow classical turbulent behavior and dissipate energy through viscosity. In the limiting case where the buoyancy scale becomes comparable to the Kolmogorov scale, the latter scaling fails and energy is completely transferred to internal waves through wave interactions. The dimensionless forms of the relevant length scales can be written as

$$
\begin{gathered}
\hat{l}_{k}=\left(\frac{1}{R e_{\tau}^{3} \hat{\varepsilon}}\right)^{1 / 4}, \\
\hat{l}_{b}=\left(\widehat{w^{\prime 2}}\right)^{1 / 2} / \hat{N}_{\mathrm{BV}}, \\
\hat{l}_{o}=\left(\hat{\varepsilon} / \hat{N}_{\mathrm{BV}}^{3}\right)^{1 / 2},
\end{gathered}
$$

and

$$
\hat{N}_{\mathrm{BV}}=\left(-R i_{\tau} \frac{\partial \hat{c}}{\partial \hat{z}}\right)^{1 / 2} .
$$

[71] Figure 16 shows the comparison of variation of the length scales as the flow transitions to Regime II. The Kolmogorov scale in general is well captured by the QE k- $\varepsilon$ model. On the other hand, the discrepancy in the Ozmidov scale is manifested in the underestimation near the TKE minimum associated with the fish trap effect. The difference in the magnitude of the two length scales given by DNS in case 5 indicates that the dissipation of energy is still dominated by turbulence, and the QE k- $\varepsilon$ model is appropriate for describing the flow and turbulence field. As the flow 
YEH ET AL.: TURBIDITY CURRENT WITH A ROOF
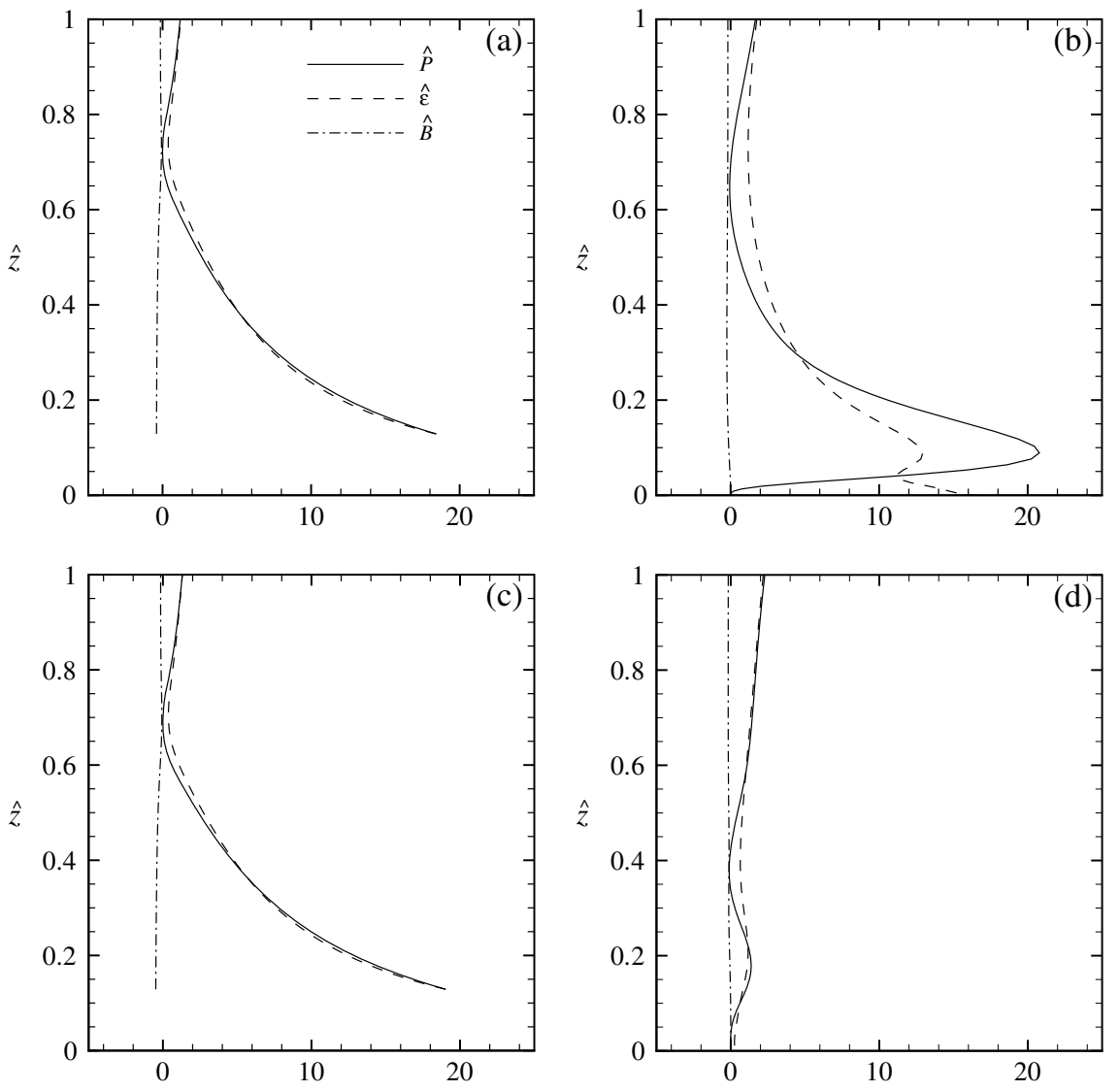

Figure 15. Energy budget profile for case $5(\mathrm{a}, \mathrm{b})$ and case $6(\mathrm{c}, \mathrm{d})$. Results from (a, c) QE k- $\varepsilon$ for the two cases are compared with $(b, d)$ DNS. Significant reduction of energy production and dissipation rate as the flow relaminarizes predicted by DNS is not captured by QE k- $\varepsilon$.
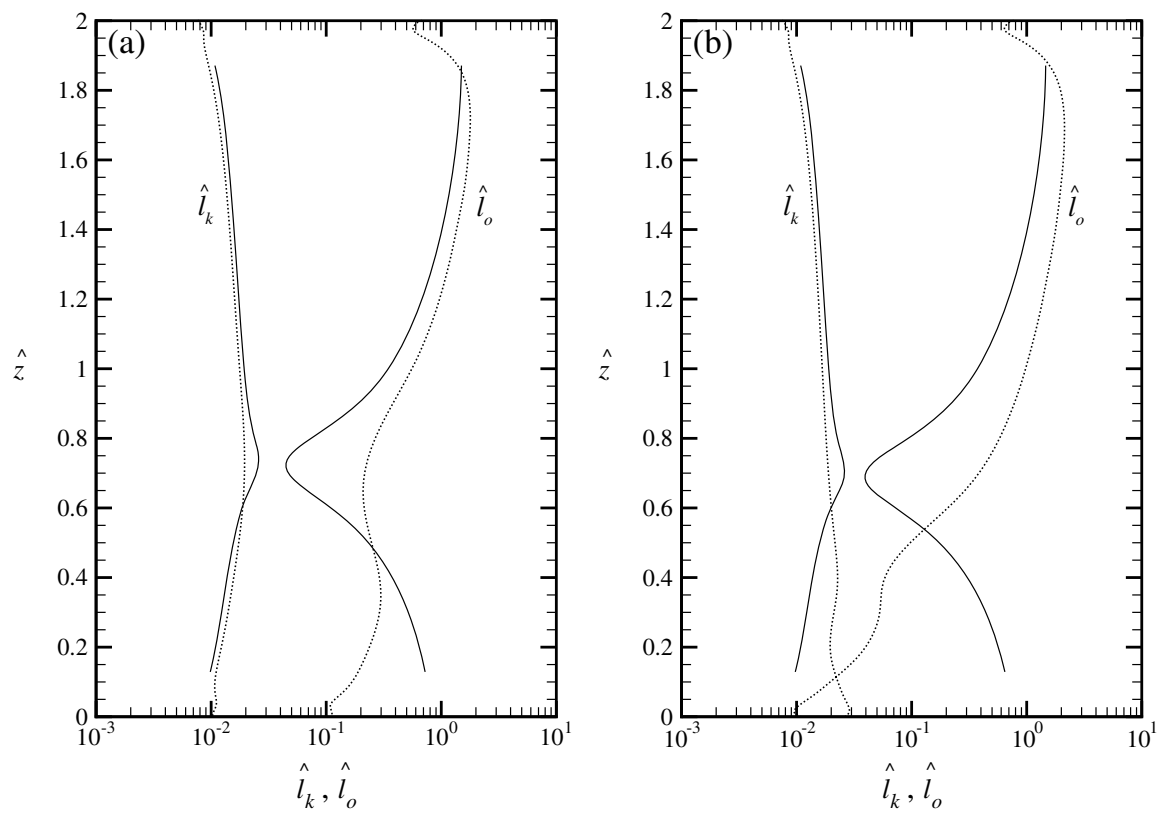

Figure 16. Comparisons of Kolmogorov length scale $\hat{l}_{k}$ and Ozmidov length scales $\hat{l}_{o}$ between QE k- $\varepsilon$ (solid line) and DNS (dotted line) for (a) case 5 (Regime I) and (b) case 6 (Regime II). 

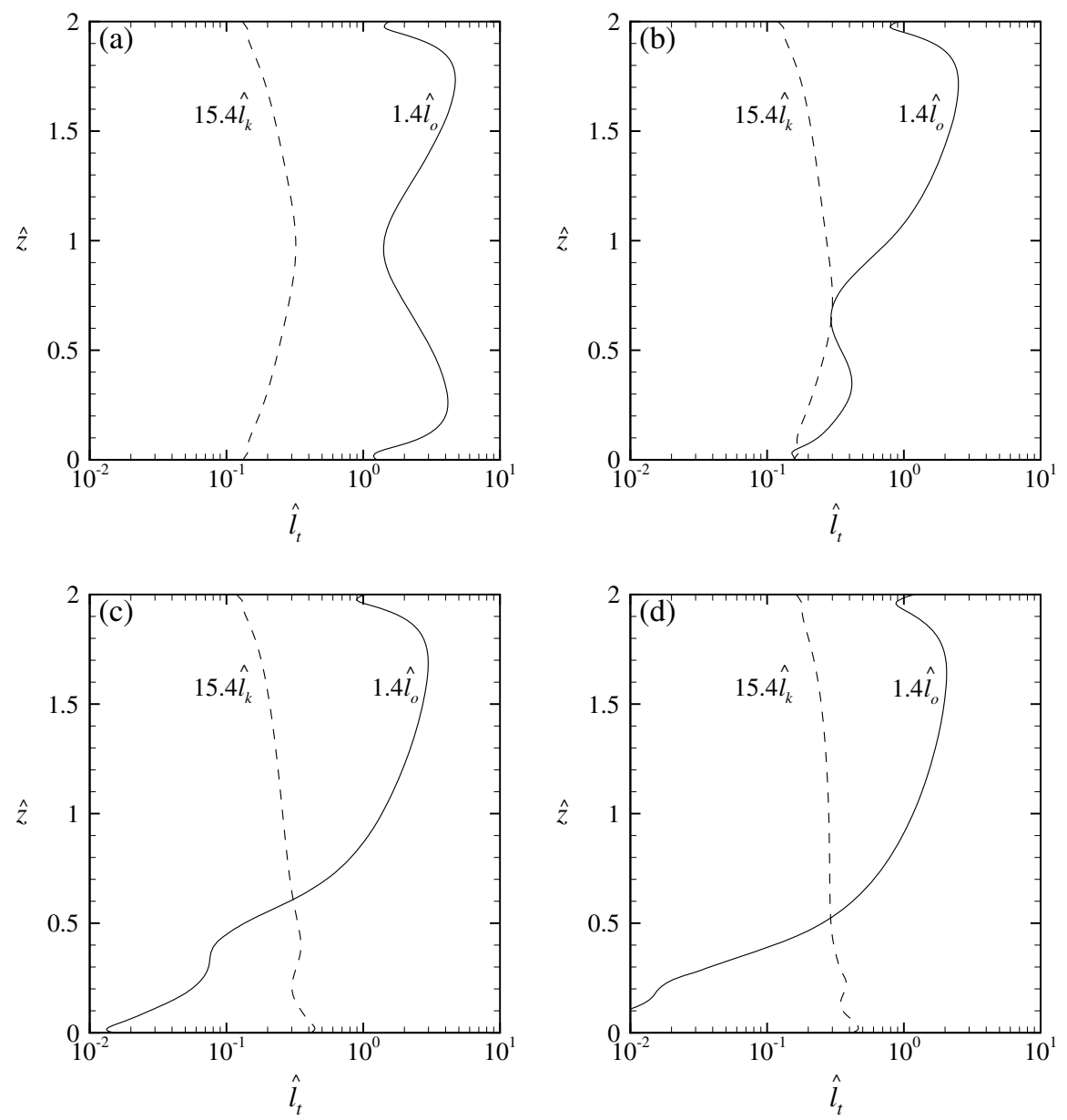

Figure 17. Comparison of the bounding length scales for (a) case 1, (b) case 5, (c) case 6, and (d) case 10.

relaminarizes, the two scales become comparable in the nearbed region. Such phenomenon however is not reflected in the length scales predicted by $\mathrm{QE} \mathrm{k}-\varepsilon$, where the dissipation of energy-dominated turbulence persists.

[72] To further examine the level of scaling failure of the RANS models as the stratification effect intensifies, we adopt the criteria proposed in the experimental studies of Stillinger et al. [1983]. In their grid turbulence experiment in a saltstratified flow, they determined the onset of the transition from a fully turbulent field to internal waves by a break in the RMS value of the density fluctuation $\left(\overline{\rho^{\prime 2}}=0\right)$ from non-stratified growth patterns. Complete transition is determined by zero Reynolds flux $\left(\overline{\rho^{\prime} w^{\prime}}=0\right)$ since waves are capable of transporting momentum but not mass. Using these characterizations, they proposed three ranges for the wavelength $\hat{l}_{t}$ of possible turbulent motions to describe energy dissipation mechanisms: $\hat{l}_{t}>1.4 \hat{l}_{o}$ corresponding to strong influence or complete dominance of internal waves; $1.4 \hat{l}_{o}>\hat{l}_{t}>15.4 \hat{l}_{k}$ corresponding to dominance of the energy cascade process over internal wave motions, and $1.4 \hat{l}_{o}>15.4 \hat{l}_{k}>\hat{l}_{t}$ corresponding to the dominance of energy dissipation by viscosity.

[73] In Figure 17, we plot for cases 1, 5, 6, and 10 the bounding length scales and the corresponding three ranges, as computed using the results of DNS. It can be seen in case 1 that $1.4 \hat{l}_{o}>15.4 \hat{l}_{k}$ is satisfied everywhere in the vertical.
However, as stratification effects increase, the buoyancyrelated scale becomes comparable to or less than $15.4 \hat{l}_{k}$ in a significant portion of the flow. Within such a region, a part of the turbulent field is likely to dissipate energy through internal wave motions. Note that the onset of this breakdown occurs in case 5, before flow relaminarization is even observed. In this case, overall agreement between RANS and DNS is found in the velocity profile (Figure $5 \mathrm{c}$ ), but RANS predictions deviate from DNS below the velocity maximum, a region where the magnitude of two length scales becomes comparable. Comparison with Figures $5 \mathrm{c}$ and $6 \mathrm{c}$ further suggest that the turbulence models perform reasonably well in the region where the condition $1.4 \hat{l}_{o} \gg 15.4 \hat{l}_{k}$ is satisfied. As the flow relaminarizes, the Ozmidov length scale is greatly reduced in the near-bed region, which indicates that internal wave motions are likely the main mechanism for energy dissipation. However, due to the increasing bias of the concentration profile toward the bed, the extent of such a region weakly decreases with increasing $\hat{v}_{s}$ (Figures $17 \mathrm{c}$ and $17 \mathrm{~d}$ ).

[74] The analysis presented here of the DNS results of C09 shows that the flow makes the transition from viscous dissipation to transfer of energy to internal waves as $\hat{v}_{s}$ increases beyond a value of 0.022 . More specifically, flows in Regime II are characterized by the complete dominance by internal waves in the lower water column, resulting in the failure of the RANS model predictions. In principle, separate 

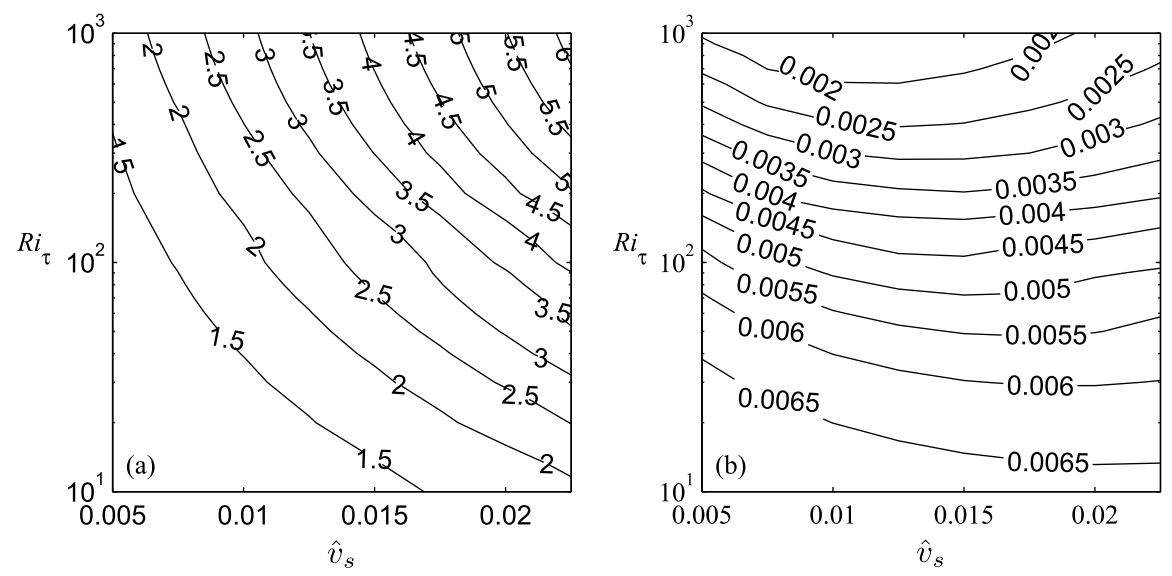

Figure 18. Variation of (a) $r_{0}$ and (b) $C_{f}$ as functions of $\hat{v}_{s}$ and $R i_{\tau}$. The QE k- $\varepsilon$ model has been applied in all simulations.

models are required to describe these two types of flows [D'Asaro and Lien, 2000]: (a) stratified turbulence models such as the RANS models presented in this study, which adequately describe the turbulent field under weakly stratified conditions and (b) wave-wave interaction models [e.g., Müller et al., 1986] which account for internal wave motion for strongly stratified flows. The use of conventional RANS models to describe the energy leakage from TKE to internal waves requires reparameterization and special closures [e. g., Baumert and Peters, 2004]. However, as pointed out by Baumert and Peters [2004] and Baumert [2012b], the key issue behind using such models to capture the wave motions is possibly the standard two-way split approach of Reynolds decomposition, which does not isolate the periodic oscillation from the fluctuation term. By including a wave component in the decomposition and treating both vortices and waves as colliding particles [Baumert, 2012a], Baumert [2012b] proposed a three-equation model which describes the energy balance in the coexistence of mean flow, turbulence, and internal waves. Such a model, though still requiring further validation, may serve to bridge the gap between the two mechanisms and enhance our progress toward a unified theory.

\section{Effect of Stratification on Flow Characteristics of Turbidity Currents}

[75] An important factor in modeling large-scale turbidity currents and the resulting morphodynamics using layer-averaged models is the characterization of several key flow parameters such as the ratio of near-bed concentration to layer-averaged concentration $r_{0}$ and the bed resistance coefficient $C_{f}$ [e.g., Fildani et al., 2006]. Empirical documentation of these parameters is lacking, mainly due to the previously described difficulties in the measurement of real-time events and scale effects in laboratory experiments. In practice, applications of layer-averaged formulations for turbidity currents often invoke values for these parameters based on open channel flows under carrying suspended sediment under uniform conditions. However, it is clear that we cannot apply these values with confidence, since the two types of flows differ significantly in their internal structure.

[76] In this section, we provide a turbidity current based analysis to provide predictive relations for $r_{0}$ and $C_{f}$, using results from the configuration of TCR. Here we specifically define these parameters as

$$
\begin{gathered}
r_{0}=\hat{c}_{b}=\frac{c_{b}}{C} \\
C_{f}=\frac{u_{*_{,}}^{2}+u_{*, b}^{2}}{U^{2}} .
\end{gathered}
$$

[77] We assume that the threshold-settling velocity for flow to relaminarize is universal at $\hat{v}_{s} \approx 0.022$ (it should be noted, however, that results obtained later by the authors of C09 show that this value increases weakly with increasing shear Reynolds number and decreasing shear Richardson number). For comparison purposes, we also retain the value of $R e_{\tau}$ of 180 . We have performed the computations necessary to quantify $r_{0}$ and $C_{f}$ and limited their computation to values of $\hat{v}_{s}$ below the threshold value. The best performing model based on the analysis above, i.e., the QE k- $\varepsilon$ model, is used here for all calculations.

[78] The dependency of $r_{0}$ and $C_{f}$ on $\hat{v}_{s}$ and $R i_{\tau}$ is examined in Figure 18. Figure 18a shows that the value of $r_{0}$ increases with both $\hat{v}_{S}$ and $R i_{\tau}$. This demonstrates that both parameters increase the effect of density stratification and cause the concentration profile to become less uniform. Increase in the value of $\hat{v}_{S}$, which directly describes the tendency for the sediment to settle, is found to be more effective in biasing the profile than increase in $R i_{\tau}$. On the other hand, the change in $C_{f}$ with increasing $\hat{v}_{s}$ is not monotonic. At lower values of $\hat{v}_{s}$, increasing stratification effects tend to reduce $C_{f}$, and thus enhance the flow velocity due to the higher driving force in the lower part of the channel. As $\hat{v}_{s}$ increases and the sediment concentration becomes more and more biased toward the bed, $C_{f}$ increases due to the loss of the driving force in major part of the channel. The values of $C_{f}$ are found to be lower than those reported in C09. This discrepancy is mainly due to omission of the low-velocity region in the calculation of $U$, a problem which can be avoided for sufficiently large $R e_{\tau}$ and small $\hat{b}$.

[79] For turbidity currents in nature, the internal structure is also affected by the composition of the grain sizes in the flow. Fine material which requires a significant time to settle in still 

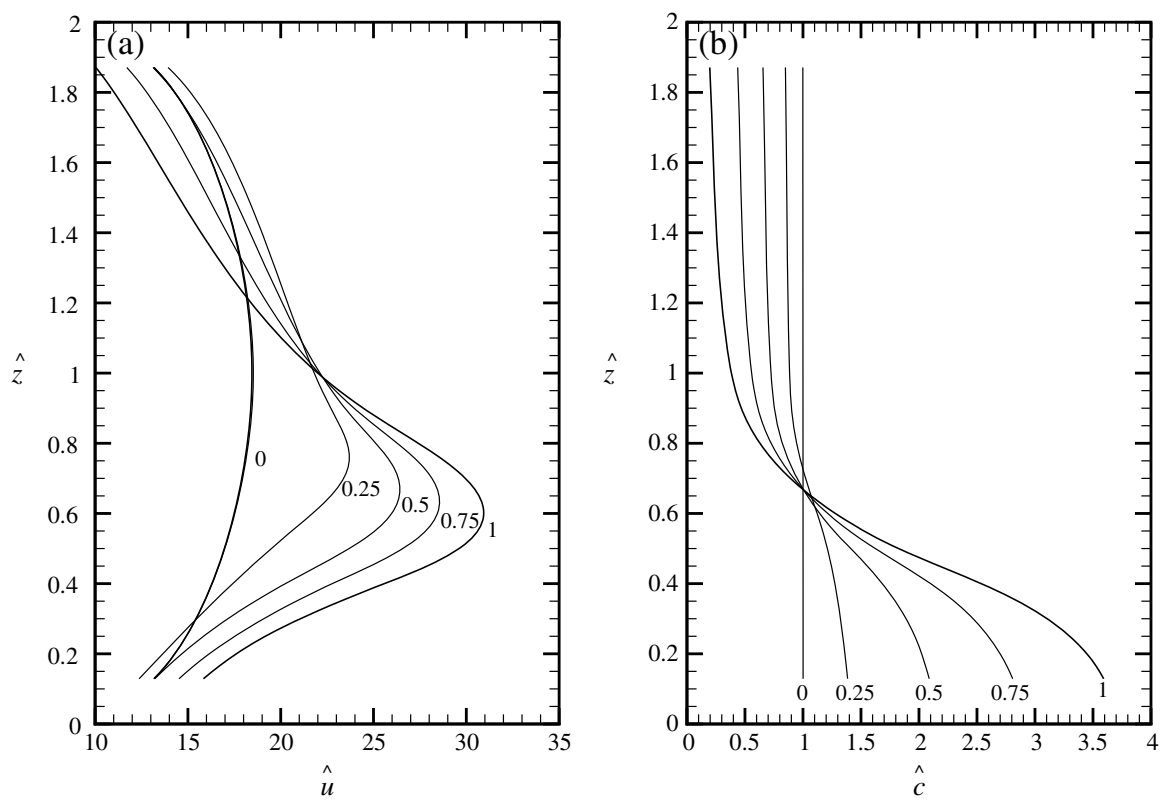

Figure 19. Variation in (a) velocity profile and (b) concentration profile due to different ratios of coarse and fine material. The numbers adjacent to the solid lines indicate the ratio of coarse to total material. The dotted lines, which are almost identical with the zero coarse material lines, indicate the velocity profile under neutral conditions. The QE k- $\varepsilon$ model has been applied in all simulations.

water tends to lead to a relatively uniform concentration profile, so reducing the overall density gradient and stratification effects compared to coarser material. Here we examine these effects using a mixture of two grain sizes. The dimensionless settling velocity $\hat{v}_{s}$ is set to 0.02 for the coarse material and 0.0001 for the fine material. The value of $R i_{\tau}$ is set to 100 to accentuate the differences among different ratios of coarse to fine material. The calculations were again done with the QE k- $\varepsilon$ closure model.

[80] Figure 19 shows velocity and total (including both sizes) volume suspended sediment concentration profiles for ratios of coarse to total material varying from 0 to 1 . The figure shows that the velocity and concentration profiles differ significantly as the ratio changes. With increase of the finer material in the flow, stratification effects weaken and the mean flow characteristics approach those under neutral conditions. Such a transition is accompanied by decrease in the near-bed concentration and increase in the resistance coefficient due to the reduction in depth-averaged velocity $U$. The results shown here have demonstrated, along with previous results due to Salaheldin et al. [2000], that fine material plays an important role in sustaining turbulence. This turbulence in turn keeps the coarse as well as fine material in suspension. Therefore, a current with a sediment mixture is likely to travel a much farther distance than that with only coarser part of that mixture. Owing to this reason, natural turbidity currents containing a sufficiently large fraction of mud can travel hundreds of kilometers into the submarine environment before dissipating, so emplacing long submarine channels [Babonneau et al., 2002; Spinewine et al., 2011].

[81] Results from Figures 18 and 19 demonstrate the variability of the parameters as a turbidity current evolves downstream. They are important in understanding key features such as upward and downstream fining of the deposit.
However, care must be taken when extrapolating these results to natural flows. First of all, the presence of a roof creates turbulence, resulting in a local TKE maximum which is comparable to that at bed. Thus, in a way, the results presented in this study is most applicable to natural turbidity currents under Froude-supercritical conditions [see e.g., Sequeiros et al., 2010], where turbulence is created by the flow entrainment at the interface of the current and the ambient fluid. However, the effects of flow entrainment on the flow structure and parameters, which is prohibited by TCR, have not been fully understood. Second, the effect of the depth-averaged concentration $C$ in the TCR formulation does not affect the level of stratification and flow structure. For natural turbidity currents, however, the effects of total sediment concentration can further modulate turbulence and consequently influence morphordynamic processes. In particular, high sediment concentration leads to hindered settling and turbulence damping. This results in rapid deposition without grain sorting in the lower part of the deposit [Lowe, 1982; Baas et al., 2004]. Such a mechanism, as opposed to flow relaminarization due to self-stratification [Cantero et al., 2012], has been one of the main hypotheses for the structureless deposit found in the base of the Bouma sequence [Bouma, 1962]. The current RANS models, however, do not account for either mechanism. In order to characterize the variability of the parameters for natural turbidity currents, the effects of the above factors on the flow structure must be understood to a better extent.

\section{Conclusion}

[82] Using the configuration of a turbidity current with a roof (TCR), we examined the capability of Reynolds-averaged Navier-Stokes (RANS) models to capture the internal structure 
of turbidity currents under conditions of self-stratification by suspended sediment. Three turbulence closures, i.e., the Mellor-Yamada (M-Y) scheme, the standard k- $\varepsilon$ scheme, and the quasi-equilibrium $\mathrm{k}-\varepsilon(\mathrm{QE} \mathrm{k}-\varepsilon)$ scheme, were implemented and compared with the results of direct numerical simulation (DNS) using the cases studied by C09.

[83] The DNS results of C09 studied the flow in the TCR configuration, for which the shear Richardson number $R i_{\tau}$ is held constant at 11.4 but dimensionless fall velocity $\hat{v}_{S}$ is increased. They found that for a dimensionless fall velocity below about 0.022 , increasing $\hat{v}_{s}$ resulted in increasing stratification effects, with gradual damping of the turbulence in the lower part of the flow. Here this regime is called Regime I. Values of $\hat{v}_{s}$ in excess of 0.022 , i.e., Regime II, corresponded to a radical modification of the flow, with stratification effects so strong that turbulence is nearly or completely extinguished in a substantial region near the bed. Cantero et al. [2012] argued that such flows are unsustainable in nature. This is because suspended sediment would rain out without resuspension. The flow would thus lose its driving force and die. Cantero et al. [2012] showed that the abrupt transition from Regime I to Regime II can be mediated by either declining slope or increasing width in the downstream direction.

[84] We show that for Regime I flow, both M-Y and QE k- $\varepsilon$ perform reasonably well in describing the mean flow and turbulent characteristics computed from DNS. As the level of stratification increases, however, increasing deviation from DNS is found in the region below the velocity maximum. The standard $\mathrm{k}-\varepsilon$ model, on the other hand, consistently underestimates stratification effects. This result implies that the choice of the stability functions may play a more important role than the choice of the closure equations used in these two-equation models.

[85] In fact, the structural similarity inherent to the most commonly used two-equation models has been demonstrated in previous studies [Umlauf and Burchard, 2003; Kantha, 2004]. It has been shown that all these models belong to a family with a generic structure consisting of a TKE equation and a length scale equation. While the comparison and selection of the optimal two-equation model for the present problem is not the main purpose of this study, the similarity in structure is at the root of two failings of the DNS models: the "fish trap" effect within Regime I and the collapse of near-bed turbulence within Regime II.

[86] The fish trap effect predicted by the RANS models is manifested in terms of an upward normal profile of suspended sediment which shows two regions, i.e., a region of relatively high suspended sediment concentration below the velocity maximum and a region of relatively low concentration above it. These regions are separated by a relatively thin zone of rapidly declining concentration. Although this zone gives the appearance of a lutocline, comparison with DNS reveals that it is an artifact of the generic structure of all three RANS, which force the Reynolds stress to vanish precisely at the point of velocity maximum. Neither this coincidence nor the apparent lutocline were observed in the DNS simulations.

[87] The appearance of the fish trap effect in the RANS models places limitations on their application to field-scale turbidity currents. Under conditions of net sediment entrainment from the bed, the fish trap effect tends to sequester the suspended sediment below the velocity maximum. This incomplete mixing can be expected to affect the downstream evolution of the current. It may be possible to overcome this limitation by using a more advanced closure scheme, by using large-eddy simulation [Armenio and Sarkar, 2002; Taylor et al., 2005], or by considering convective flows with a vertical component that are likely to occur in the field [Straub et al., 2011].

[88] Within Regime II, i.e., beyond the threshold $\hat{v}_{S}$ $=0.022$, all three RANS models fail to capture the internal structure of the flow. In particular, they fail to capture the phenomenon of near or complete extinction of near-bed turbulence. This failure is due in part to the inability of the boundary conditions classically used in such models to accurately describe the flow. But the result that the failure is not remedied by forcing the flow to obey boundary conditions obtained from DNS indicates that the models themselves are insufficient to describe strong stratification effects.

[89] The RANS models used here are then further limited in field applicability, in that they can be used neither to describe the fate of a flow when near-bed turbulence collapses nor to characterize the type of sediment deposit they would emplace. We relate this failure to the inability of the RANS models to describe a transition from turbulence to internal waves under conditions of strong stratification.

[90] The standard hypothesis of RANS models is that energy is dissipated by the eddies at the fine scale of the turbulence and that stratification effects simply modify this process. Under conditions of sufficiently high stratification, however, the vertical density gradient is so strong that vertical turbulent fluctuations are greatly suppressed. Near-horizontal fluctuations then transfer energy to internal waves. We show that (a) the DNS predictions in Regime II are in the range for transfer to internal waves and (b) the RANS models do not capture this transfer. We suggest that a model which can describe the transition of energy dissipation from the classical cascade model to full wave interaction is required to capture the flow characteristics under extreme stratified conditions and, in particular, the phenomenon of collapse of near-bed turbulence.

[91] The internal structure of a turbidity current is highly complex, particularly when the interactions between the current and bedforms on the bed (such as dunes, antidunes, or bars) become important. We should note that both the flow configuration (TCR) and the benchmark tool (DNS) used in this study are subject to several assumptions and simplifications, and the model results thus do not account for the full complexity of natural turbidity currents. Having said that, the TCR methodology presented here allows for a focused study of the effect of stratification on the internal structure and flow characteristics of turbidity currents. Moreover, the use of the TCR configuration using both RANS and DNS yields valuable insight on the limitations of RANS turbulence closures in modeling stratified flows. The results obtained here cannot be directly translated to turbidity currents in nature, because the presence of a roof precludes entrainment of ambient water at the upper interface. They do, however, provide the basis for future studies on more refined parameterization for field-scale modeling of turbidity currents.

\section{Appendix A}

[92] Under fully relaminarized flow conditions, analytical solutions can be derived using the same approach described in C09, but with the boundary conditions as given in section 2.3. The results are summarized below. 
[93] The dimensionless velocity and sediment concentration profiles are given by

$$
\hat{u}=p_{1}\left[\exp \left(-\hat{v}_{S} \operatorname{Re}_{\tau} S c(\hat{z}-1)\right)+p_{2} \hat{z}+p_{3}\right]
$$

and

$$
\hat{c}=\frac{-\hat{v}_{s} \operatorname{Re} \operatorname{Se}_{\tau}(1-\hat{b})}{\sinh \left(-\hat{v}_{S} \operatorname{Re}_{\tau} S c(1-\hat{b})\right)} \exp \left(-\hat{v}_{s} \operatorname{Re} e_{\tau} S c(\hat{z}-1)\right),
$$

where

and

$$
\begin{gathered}
p_{1}=\frac{\operatorname{Re}_{\tau}(1-\hat{b})}{\hat{v}_{S} \operatorname{Re}_{\tau} S c \sinh \left(-\hat{v}_{s} \operatorname{Re} e_{\tau} S c(1-\hat{b})\right)}, \\
p_{2}=\frac{-2 \sinh \left(-\hat{v}_{s} \operatorname{Re}_{\tau} S c(1-\hat{b})\right)+\left(\hat{u}_{t}-\hat{u}_{b}\right) / p_{1}}{2(1-\hat{b})},
\end{gathered}
$$

$$
p_{3}=\frac{\hat{u}_{b}}{p_{1}}-\exp \left(-\hat{v}_{s} \operatorname{Re} \tau s c(\hat{b}-1)\right)-p_{2} \hat{b}
$$

\section{Notation}

$$
\begin{aligned}
& \alpha \quad \text { empirical constant } \\
& \varepsilon \quad \text { energy dissipation rate }\left[L^{2} / T^{3}\right] \\
& \hat{\varepsilon} \text { dimensionless energy dissipation rate } \\
& \kappa \quad \text { von Kármán constant } \\
& v \text { kinematic viscosity }\left[L^{2} / T\right] \\
& v_{c} \quad \text { molecular diffusivity }\left[L^{2} / T\right] \\
& \hat{v}_{t} \quad \text { dimensionless eddy viscosity } \\
& \hat{v}_{t 0} \quad \text { dimensionless eddy viscosity } \\
& \text { under neutral conditions } \\
& \hat{v}_{t c} \quad \text { dimensionless eddy diffusivity } \\
& \hat{v}_{t c 0} \quad \text { dimensionless eddy diffusivity under } \\
& \text { neutral conditions } \\
& \rho_{a} \quad \text { density of ambient fluid }\left[M / L^{3}\right] \\
& \rho_{s} \quad \text { density of sediment }\left[M / L^{3}\right] \\
& \sigma_{\varepsilon}, \sigma_{k} \text { model coefficients } \\
& \tau \text { total mean shear stress }\left[M / L / T^{2}\right] \\
& \tau_{v} \quad \text { viscous stress }\left[M / L / T^{2}\right] \\
& \tau_{R e} \quad \text { Reynolds stress }\left[M / L / T^{2}\right] \\
& \tau_{b} \quad \text { total mean shear stress on the bed }\left[M / L / T^{2}\right] \\
& \tau_{t} \quad \text { total mean shear stress on the roof }\left[M / L / T^{2}\right] \\
& A_{1}, A_{2} \quad \text { model coefficients } \\
& \hat{B} \text { dimensionless buoyancy production rate } \\
& B_{1}, B_{2} \quad \text { model coefficients } \\
& \hat{B}_{b} \text { near-bed buoyancy production rate } \\
& \hat{B}_{t} \text { near-roof buoyancy production rate } \\
& b \text { reference height }[L] \\
& \hat{b} \text { dimensionless reference height } \\
& \text { C depth-averaged sediment volume } \\
& \text { concentration } \\
& C_{\varepsilon 1}, C_{\varepsilon 2}, C_{\varepsilon 3} \quad \text { model coefficients } \\
& C_{\mu}, C_{\mu c} \quad \text { model coefficients } \\
& C_{f} \text { resistance coefficient } \\
& c \text { Reynolds-averaged total sediment volume }
\end{aligned}
$$

$\hat{c}$ normalized Reynolds-averaged total sediment volume concentration

$c_{\mu}, c_{\mu c}$ stability functions

$c_{\mu}^{0} \quad$ empirical constant

$c_{b}$ near-bed sediment volume concentration

$\hat{c}_{b}$ normalized near-bed sediment volume concentration

$c_{i}$ sediment volume concentration in the $i$ th size fraction

$\hat{c}_{i} \quad$ normalized sediment volume concentration in the $i$ th size fraction

DE damping efficiency

$d \hat{t}$ dimensionless time step

$d \hat{z}$ dimensionless grid size

$E_{1}, E_{2}, E_{3}$ model coefficients

$F_{v}$ viscous flux $[L / T]$

$F_{R e}$ Reynolds flux $[L / T]$

$g$ gravitational acceleration $\left[L / T^{2}\right]$

$h$ half roof height $[L]$

$k$ turbulent kinetic energy $\left[L^{2} / T^{2}\right]$

$\hat{k}$ dimensionless turbulent kinetic energy

$\hat{L}$ measure of distance from the wall $(=\max (\hat{z}, 2-\hat{z}))$

$\hat{l}$ dimensionless master length scale

$l_{b}$ buoyancy length scale $[L]$

$\hat{l}_{b}$ dimensionless buoyancy length scale

$l_{k} \quad$ Kolmogorov length scale $[L]$

$\hat{l}_{k}$ dimensionless Kolmogorov length scale

$l_{o}$ Ozmidov length scale $[L]$

$\hat{l}_{o}$ dimensionless Ozmidov length scale

$\hat{l}_{t}$ dimensionless wavelength for turbulent motions

$N$ total number of sediment size fractions

$N_{\mathrm{BV}} \quad$ Brunt-Väisälä frequency [1/T]

$\hat{N}_{\text {BV }}$ dimensionless Brunt-Väisälä frequency

$\hat{P}$ dimensionless energy production rate

$p$ Reynolds-averaged total pressure $\left[M / L / T^{2}\right]$

$p_{i}^{\mathrm{wc}}$ volumetric proportion of the sediment in the $i$ th fraction range

$\hat{q}$ measure of dimensionless turbulent kinetic energy per unit mass $(=\sqrt{2 \hat{k}})$

$R$ submerged specific gravity

$R e_{\tau}$ shear Reynolds number

$R i_{\tau}$ shear Richardson number

$r_{0}$ near-bed sediment volume concentration ratio

$S_{0}$ channel slope

$S_{H}, S_{M}$ stability functions

$S_{l}, S_{q}$ model coefficients

Sc Schmidt number

$S c_{t}$ turbulent Schmidt number

$t$ time [T]

$\hat{t}$ dimensionless time

$U$ depth-averaged streamwise velocity $[L / T]$

$u$ Reynolds-averaged streamwise velocity $[L / T]$

$\hat{u}$ dimensionless streamwise velocity

$u^{\prime}$ streamwise velocity fluctuation $[L / T]$ 


\begin{tabular}{|c|c|}
\hline$\overline{u^{\prime 2}}$ & streamwise Reynolds normal stress $\times(-1)\left[L^{2} / T^{2}\right]$ \\
\hline$u_{* \text {,avg }}$ & nominal shear velocity $[L / T]$ \\
\hline$u *, b$ & shear velocity at the bed $[L / T]$ \\
\hline$\hat{u} *, b$ & dimensionless shear velocity at the bed \\
\hline$u_{*, t}$ & shear velocity at the roof $[L / T]$ \\
\hline $\begin{aligned} \hat{u} *, t \\
\hat{u}_{b}\end{aligned}$ & $\begin{array}{l}\text { dimensionless shear velocity at the roof } \\
\text { dimensionless velocity at the bed }\end{array}$ \\
\hline$\hat{u}_{t}$ & dimensionless velocity at the roof \\
\hline$\overline{u^{\prime} w^{\prime}}$ & Reynolds shear stress $\times(-1)\left[L^{2} / T^{2}\right]$ \\
\hline$\widehat{\overline{u^{\prime} w^{\prime}}}$ & $\begin{array}{l}\text { dimensionless Reynolds shear } \\
\text { stress } \times(-1)\end{array}$ \\
\hline$v$ & Reynolds-averaged transverse velocity $[L / T]$ \\
\hline$v_{\mathrm{si}}$ & settling velocity of the $i$ th size fraction $[L / T]$ \\
\hline$\hat{v}_{\mathrm{si}}$ & $\begin{array}{l}\text { dimensionless settling velocity of the } i \text { th size } \\
\text { fraction }\end{array}$ \\
\hline$w$ & $\begin{array}{l}\text { Reynolds-averaged upward normal velocity } \\
{[L / T]}\end{array}$ \\
\hline$w^{\prime}$ & upward normal velocity fluctuation $[L / T]$ \\
\hline$\overline{w^{\prime 2}}$ & $\begin{array}{l}\text { upward normal Reynolds normal stress } \times(-1) \\
{\left[L^{2} / T^{2}\right]}\end{array}$ \\
\hline$\overline{w^{\prime} c_{i}^{\prime}}$ & $\begin{array}{l}\text { Reynolds flux } \times(-1) \text { of the } i \text { th size fraction } \\
{[L / T]}\end{array}$ \\
\hline$\widehat{\widehat{w^{\prime} c^{\prime}}}$ & $\begin{array}{l}\text { dimensionless Reynolds flux } \times(-1) \text { of the } i \text { th size } \\
\text { fraction }\end{array}$ \\
\hline$\widehat{\widehat{w^{\prime} c^{\prime}}}$ & dimensionless total Reynolds flux $\times(-1)$ \\
\hline$x$ & streamwise coordinate $[L]$ \\
\hline$y$ & transverse coordinate $[L]$ \\
\hline$z$ & upward normal coordinate $[L]$ \\
\hline$\hat{z}$ & dimensionless upward normal coordinate \\
\hline$z^{+}$ & wall unit \\
\hline$z_{f}$ & free surface elevation $[L]$ \\
\hline$J$ & dimensionless elevation for velocity maximum \\
\hline
\end{tabular}

[94] Acknowledgment. This research was funded by Shell Oil Company. We thank Jasim Imran for coining the term "fish trap" used in this study.

\section{References}

Abd El-Gawad, S., A. Cantelli, C. Pirmez, D. Minisini, Z. Sylvester, and J. Imran (2012a), Three-dimensional numerical simulation of turbidity currents in a submarine channel on the seafloor of the Niger Delta slope, J. Geophys. Res., 117, C05026, doi:10.1029/2011JC007538.

Abd El-Gawad, S., C. Pirmez, A. Cantelli, D. Minisini, Z. Sylvester, and J. Imran (2012b), 3-D numerical simulation of turbidity currents in submarine canyons off the Niger Delta, Mar. Geol., 326-328, 55-66, doi:10.1016/j.margeo.2012.06.003.

Armenio, V., and S. Sarkar (2002), An investigation of stably stratified turbulent channel flow using large-eddy simulation, J. Fluid Mech., 459, 1-42, doi:10.1017/S0022112002007851.

Baas, J. H., W. van Kestern, and G. Postma (2004), Deposits of depletive high-density turbidity currents: A flume analogue of bed geometry, structure and texture, Sedimentology, 51,1053-1088.

Babonneau, N., B. Savoye, M. Cremer, and B. Klein (2002), Morphology and architecture of the present canyon and channel system of the Zaire deep-sea fan, Mar. Pet. Geol., 19, 445-467, doi:10.1016/S0264-8172(02)00009-0.

Baumert, H. (2012a), Universal equations and constants of turbulent motion, arXiv:1203.5042v2 [physics.flu-dyn].

Baumert, H. (2012b), Turbulent mixing driven by mean-flow shear and internal gravity waves in oceans and atmospheres, arXiv:1207.1633v1 [physics.flu-dyn].

Baumert, H., and H. Peters (2000), Second-moment closures and length scales for weakly stratified turbulent shear flows, J. Geophys. Res., 105(C3), 6453-6468, doi:10.1029/1999JC900329.

Baumert, H., and H. Peters (2004), Turbulence closure, steady state, and collapse into waves, J. Phys. Oceanogr., 34, 505-512.

Bombardelli, F. A., and S. K. Jha (2009), Hierarchical modeling of the dilute transport of suspended sediment in open channels, Env. Fluid Mech., 9, 207-235, doi:10.1007/s10652-008-9091-6.
Bouma, A. (1962), Sedimentology of Some Flysch Deposits: A Graphic Approach to Facies Interpretation, Elsevier, Amsterdam.

Bradford, S. F., and N. D. Katopodes (1999), Hydrodynamics of turbidity underflows. I: Formulation and numerical analysis, J. Hydraul. Eng., 125(10), 1006-1015.

Burchard, H. (2001), On the q21 equation by Mellor and Yamada (1982), J. Phys. Oceanogr., 31(5), 1377-1387, doi:10.1175/1520-0485(2001) $031<1377$ :OTQLEB > 2.0.CO;2.

Burchard, H., and H. Baumert (1995), On the performance of a mixed-layer model based on the k-e turbulence closure, J. Geophys. Res., 100(C5), 8523-8540, doi:10.1029/94JC03229.

Burchard, H., O. Petersen, and T. P. Rippeth (1998), Comparing the performance of the Mellor-Yamada and the k- $\varepsilon$ two-equation turbulence models, J. Geophys. Res., 103(C5), 10,543-10,554, doi:10.1029/ 98JC00261.

Cantero, M. I., S. Balachandar, A. Cantelli, C. Primez, and G. Parker (2009), Turbidity current with a roof: Direct numerical simulation of self-stratified turbulent channel flow driven by suspended sediment, J. Geophys. Res., 114, C03008, doi:10.1029/2008JC004978.

Cantero, M. I., A. Cantelli, C. Pirmez, S. Balachandar, D. Mohrig, T. A. Hickson, T. Yeh, H. Naruse, and G. Parker (2012), Emplacement of massive turbidites linked to extinction of turbulence in turbidity currents, Nat. Geosci., 5, 42-45, doi:10.1038/ngeo1320.

Cellino, M., and W. H. Graf (1999), Sediment-laden flow in open-channels under noncapacity and capacity conditions, J. Hydraul. Eng., 125(5), 455-462, doi:10.1061/(ASCE)0733-9429(1999)125:5(455).

Choi, S. U., and M. H. Garcia (2002), k- $\varepsilon$ turbulence modeling of turbidity currents developing two dimensionally on a slope, J. Hydraul. Eng., 128(1), 55-63, doi:10.1061/(ASCE)0733-9429(2002)128:1(55).

Coleman, N. L. (1981), Velocity profiles with suspended sediment, J. Hydraul. Res., 19(3), 211-229, doi:10.1080/00221688109499516.

Coleman, N. L. (1986), Effects of suspended sediment on the open-channel velocity distribution, Water Resour. Res., 22(10), 1377-1384, doi:10.1029/WR022i010p01377.

D'Asaro, E. A., and R. C. Lien (2000), The wave-turbulence transition for stratified flows, J. Phys. Oceanogr., 30, 1669-1678, doi:10.1175/15200485(2000)030<1669:TWTTFS $>2.0$.CO; 2 .

Einstein, H. A., and N. Chien (1955), Effects of Heavy Sediment Concentration Near the Bed on Velocity and Sediment Distribution, MRD Sediment Series No. 8, University of California at Berkeley, Institute of Engineering Research, Berkeley, CA, pp. 76.

Ellison, T. H., and J. S. Turner (1959), Turbulence entrainment in stratified flows, J. Fluid Mech., 6, 423-448, doi:10.1017/S0022112059000738.

Fildani, A., W. R. Normark, S. Kostic, and G. Parker (2006), Channel formation by flow stripping: Large-scale scour features along the Monterey East Channel and their relation to sediment waves, Sedimentology, 53(6), 1265-1287, doi:10.1111/j.1365-3091.2006.00812.x.

Galperin, B., L. H. Kantha, S. Hassid, and A. Rosati (1988), A quasi-equilibrium turbulent energy model for geophysical flows, J. Atmos. Sci., 45(1), 55-62, doi:10.1175/1520-0469(1988)045 < 0055:AQETEM > 2.0.CO;2.

Garcia, M. H., and G. Parker (1991), Entrainment of bed sediment into suspension, J. Hydaul. Eng., 117(4), 414-435, doi:10.1061/(ASCE)07339429(1991)117:4(414).

Gore, R. A., and C. T. Crowe (1991), Modulation of turbulence by a dispersed phase, J. Fluid Eng., 113(2), 304-307, doi:10.1115/1.2909497.

Gregg, M. C. (1987), Diapycnal mixing in the thermocline: A review, J. Geophys. Res., 92(C5), 5249-5286, doi:10.1029/JC092iC05p05249.

Gregg, M. C. (1989), Scaling of turbulent dissipation in the thermocline, J. Geophys. Res., 94(C7), 9686-9698, doi:10.1029/JC094iC07p09686.

Huang, H., J. Imran, and C. Pirmez (2004), Numerical model of turbidity currents with a deforming bottom boundary, J. Hydraul. Eng., 131(4), 283-293, doi:10.1061/(ASCE)0733-9429(2005)131:4(283).

Imran, J., G. Parker, and N. Katopodes (1998), A numerical model of channel inception on submarine fans, J. Geophys. Res., 103(C1), 1219-1238, doi:10.1029/97JC01721.

Ito, M., and T. Saito (2006), Gravel waves in an ancient canyon: Analogous features and formative processes of coarse-grained bedforms in a submarine-fan system, the Lower Pleistocene of the Boso Peninsula, Japan, J. Sediment. Res., 76(12), 1274-1283, doi:10.2110/jsr.2006.093.

Kantha, L. H. (2004), The length scale equation in turbulence models, Nonlinear Processes Geophys., 11(1), 83-97, doi:10.5194/npg-11-83-2004.

Khan, S. M., and J. Imran (2008), Numerical investigation of turbidity currents flowing through minibasins on the continental slope, J. Sediment. Res., 78, 245-257, doi:10.2110/jsr.2008.031.

Kostic, S., and G. Parker (2003), Progradational sand-mud deltas in lakes and reservoirs. Part 1 . Theory and numerical modeling, J. Hydraul. Res., 41(2), 127-140, doi:10.1080/00221680309499956.

Lowe, D. R. (1982), Sediment gravity flows. 2. Depositional models with special reference to the deposits of high-density turbidity currents, J. Sediment. Res., 52, 279-298. 
Luyten, P. J., E. Deleersnijder, J. Ozer, and K. G. Ruddick (1996), Presentation of a family of turbulence closure models for stratified shallow water flows and preliminary application to the Rhine outflow region, Cont. Shelf Res., 16(1), 101-130, doi:10.1016/0278-4343(95)93591-V.

Lyn, D. A. (1988), A similarity approach to turbulent sediment-laden flows in open channels, J. Fluid Mech., 193, 1-26, doi:10.1017/S0022112088002034.

Mellor, G. L., and T. Yamada (1974), A hierarchy of turbulence closure models for planetary boundary layers, J. Atmos. Sci., 31(7), 1791-1806, doi:10.1175/1520-0469(1974)031 < 1791:AHOTCM > 2.0.CO;2.

Mellor, G. L., and T. Yamada (1982), Development of a turbulence closure model for geophysical fluid problems, Rev. Geophys., 20(4), 851-875, doi:10.1029/RG020i004p00851.

Middleton, G. V. (1993), Sediment deposition from turbidity currents, Ann. Rev. Earth Planet. Sci., 21, 89-114, doi:10.1146/annurev.ea.21.050193.000513.

Monin, A. S., and A. M. Obukhov (1954), Osnovnye zakonomernost turbulentnogo peremeshivanija $\mathrm{v}$ prizemnom sloe atmosfery (Basic laws of turbulent mixing in the atmosphere near the ground), Tr. Geofiz. Inst., Akad. Nauk SSSR, 24(151), 163-187.

Müller, P., D. J. Olbers, J. Willebrand, G. Holloway, F. Henyey, and N. Ponphrey (1986), Nonlinear interactions among internal gravity waves, Rev. Geophys., 24(3), 493-536, doi:10.1029/RG024i003p00493.

Nino, Y., and M. Garcia (1998), Engelund's analysis of turbulent energy and suspended load, J. Eng. Mech., 124, 480-483, doi:10.1061/(ASCE)07339399(1998)124:4(480).

Pantin, H. M. (1979), Interaction between velocity and effective density in turbidity flow: Phase plane analysis with criteria for autosuspension, Mar. Geol., 31, 59-99, doi:10.1016/0025-3227(79)90057-4.

Parker, G., Y. Fukushima, and H. M. Pantin (1986), Self-accelerating turbidity currents, J. Fluid Mech., 171, 145-181, doi:10.1017/S0022112086001404.

Patel, V. C., W. Rodi, and G. Scheuerer (1985), Turbulence models for near-wall and low Reynolds number flows: A review, AIAA J., 23(9), 1308-1319.

Pope, S. B. (2000), Turbulent flows, Cambridge University Press, NY.

Rodi, W. (1993), Turbulence Models and their Application in Hydraulics - A State-of-the-Art Review, A. A. Balkema Publishers, Brookfield, VT, USA, 3rd ed., pp. 104.

Salaheldin, T. M., J. Imran, M. H. Chaudhry, and C. Reed (2000), Role of fine-grained sediment in turbidity current flow dynamics and resulting deposits, Mar. Geol., 171, 21-38, doi:10.1016/S0025-3227 (00)00114-6.

Sequeiros, O. E., B. Spinewine, R. T. Beaubouef, T. Sun, M. H. Garcia, and G. Parker (2010), Characteristics of velocity and excess density profiles of saline underflows and turbidity currents flowing over a mobile bed, J. Hydraul. Eng., 136(7), 412-433, doi:10.1061/(ASCE)HY.19437900.0000200.

Spinewine, B., T. Sun, N. Babonneau, and G. Parker (2011), Self-similar long profiles of aggrading submarine leveed channels: Analytical solution and its application to the Amazon Channel, J. Geophys. Res., 116, F03004, doi:10.1029/2010JF001937.

Stillinger, D. C., K. N. Helland, and C. W. van Atta (1983), Experiments on the transition of homogeneous turbulence to internal waves in a stratified fluid, J. Fluid Mech., 131, 91-122, doi:10.1017/S0022112083001251.

Straub, K. M., D. Mohrig, J. Buttles, B. McElroy, B. McElroy, and C. Pirmez (2011), Quantifying the influence of channel sinuosity on the depositional mechanics of channelized turbidity currents: A laboratory study, Mar. Pet. Geol., 28(3), 744-760, doi:10.1016/j.marpetgeo.2010.05.014.

Taylor, J. R., S. Sarkar, and V. Armenio (2005), Large eddy simulation of stably stratified open channel flow, Phys. Fluids, 17, 116,602, doi:10.1063/1.2130747.

Turner, J. S. (1973), Buoyancy Effects in Fluids, Cambridge University Press, NY

Umlauf, L., and H. Burchard (2003), A generic length-scale equation for geophysical 959 turbulence models, J. Mar. Res., 61(2), 235-265, doi: $10.1357 / 002224003322005087$.

Vanoni, V. A. (1946), Transportation of suspended sediment by water, Am. Soc. Civ. Eng., Trans., 111, 67-133.

Versteeg H. K., and W. Malalasekera (2007), An Introduction to Computational Fluid Dynamics. The Finite Volume Method, 2nd ed. vol. 964, 503 pp, Pearson Education Limited, England.

$\mathrm{Xu}$, J. P. (2010), Normalized velocity profiles of field-measured turbidity currents, Geology, 38(6), 563-566, doi:10.1130/G30582.1.

Xu, J. P., M. A. Noble, and Rosenfeld, L. K. (2004), In-situ measurements of velocity structure within turbidity currents, Geophys. Res. Lett., 31, L09311, doi:10.1029/2004GL019718.

Yeh, T., and G. Parker (2013), Software for evaluating sediment-induced stratification in open-channel flows, Comput. Geosci., 53, 94-104, doi:10.1016/j.cageo.2011.12.004 